\title{
Fine-tuning and autoregulation of the intestinal determinant and tumor suppressor homeobox gene CDX2 by alternative splicing
}

\author{
Camille Balbinot ${ }^{1,5}$, Marie Vanier ${ }^{1,5}$, Olivier Armant ${ }^{2,6}$, Asmaa Nair ${ }^{1}$, Julien Penichon ${ }^{1}$, Christine Soret ${ }^{1}$, Elisabeth Martin ${ }^{1}$, \\ Thoueiba Saandi ${ }^{1}$, Jean-Marie Reimund ${ }^{1}$, Jacqueline Deschamps ${ }^{3}$, Felix Beck ${ }^{4}$, Claire Domon-Dell ${ }^{1}$, Isabelle Gross ${ }^{1}$, Isabelle Duluc ${ }^{1,5}$ \\ and Jean-Noël Freund ${ }^{*, 1,5}$
}

On the basis of phylogenetic analyses, we uncovered a variant of the CDX2 homeobox gene, a major regulator of the development and homeostasis of the gut epithelium, also involved in cancer. This variant, miniCDX2, is generated by alternative splicing coupled to alternative translation initiation, and contains the DNA-binding homeodomain but is devoid of transactivation domain. It is predominantly expressed in crypt cells, whereas the CDX2 protein is present in crypt cells but also in differentiated villous cells. Functional studies revealed a dominant-negative effect exerted by miniCDX2 on the transcriptional activity of CDX2, and conversely similar effects regarding several transcription-independent functions of CDX2. In addition, a regulatory role played by the CDX2 and miniCDX2 homeoproteins on their pre-mRNA splicing is displayed, through interactions with splicing factors. Overexpression of miniCDX2 in the duodenal Brunner glands leads to the expansion of the territory of these glands and ultimately to brunneroma. As a whole, this study characterized a new and original variant of the CDX2 homeobox gene. The production of this variant represents not only a novel level of regulation of this gene, but also a novel way to fine-tune its biological activity through the versatile functions exerted by the truncated variant compared to the full-length homeoprotein. This study highlights the relevance of generating protein diversity through alternative splicing in the gut and its diseases.

Cell Death and Differentiation (2017) 24, 2173-2186; doi:10.1038/cdd.2017.140; published online 1 September 2017

The intestinal epithelium is a complex cellular system in constant renewal whose dynamic homeostasis involves multiple and complementary factors and pathways. ${ }^{1,2}$ Among them, the homeotic transcription factor CDX2 has a central role during embryogenesis to determine the intestinal identity of the presumptive midgut/hindgut endoderm,, 3 and throughout adulthood for intestinal epithelium homeostasis to organize the stem cell niche, to provide tissue identity to the stem cells, and to regulate cell proliferation and differentiation. ${ }^{5-8}$ CDX2 gene expression and function need to be tightly regulated, because both loss and overexpression are lethal. ${ }^{8,9} C D X 2$ is also relevant in gut pathologies, since its strong decay in human colon cancer correlates with poor evolution, ${ }^{10,11}$ while functional studies have attributed a tumor suppressor role in the gut. ${ }^{12-15}$

Alternative splicing of pre-messenger RNA is an important facet of RNA metabolism to generate protein diversity ${ }^{16,17}$ involved in every biological process from embryonic development to tissue homeostasis, and also in diseases. ${ }^{18,19}$ However, it remains largely underrated because most of the splicing variants are only identified by their sequence without knowledge on their function. Alternative splicing is poorly documented in the gut. Here, we discovered a splicing variant of $C D X 2$ and we unveiled its original function.

\section{Results}

The CDX2 homeobox gene encodes an alternative mRNA variant, miniCDX2. To search for novel transcript isoforms in the mouse intestine, 490 million paired-end reads were generated from three independent RNA samples of the cecum. They corresponded to 16822 consistently expressed genes ( $>10$ normalized reads in all replicates), and represented $43 \%$ of all annotated genes in the mouse genome including, for instance, $98 \%$ of the intestinal stem cells markers. ${ }^{20}$ The comparison to the reference transcripts annotation identified 4799 novel possible exon-exon junctions in 1802 genes (Supplementary Table 1). This highlights the potential of alternative splicing to generate protein diversity in the gut.

Among those genes is $C D X 2$, a homeobox gene containing three exons (Figure 1A). Exploring its functional structure by RT-PCR and northern blot in human colon cancer cell lines and mouse intestine identified the variant referred to as

\footnotetext{
${ }^{1}$ Université de Strasbourg, Inserm, UMR_S1113, FMTS, Strasbourg 67000, France; ${ }^{2}$ Karlsruhe Institute of Technology, Institute of Toxicology and Genetics, Postfach 3640, Karlsruhe 76021, Germany; ${ }^{3}$ Hubrecht Institute, Developmental Biology and Stem Cell Research, Uppsalalaan 8, Utrecht 3584 CT, The Netherlands and ${ }^{4}$ Barts and The London School of Medicine and Dentistry, London E1 2ES, UK

*Corresponding author: J-N Freund, Université de Strasbourg, Inserm, UMR_S1113, FMTS, 3 Avenue Molière, Strasbourg 67200, France. Tel: +33 388277727 ; Fax: +33 3882635 38; E-mail: jean-noel.freund@ @inserm.fr

${ }^{5}$ These authors contributed equally to this work.

${ }^{6}$ Current address: Institut de Radioprotection et de Sûreté Nucléaire (IRSN), PRP-ENV/SERIS/LECO, Cadarache, Saint-Paul-lez-Durance 13115, France.

Received 18.5.17; revised 23.7.17; accepted 25.7.17; Edited by D Aberdam; published online 01.9.17
} 
A
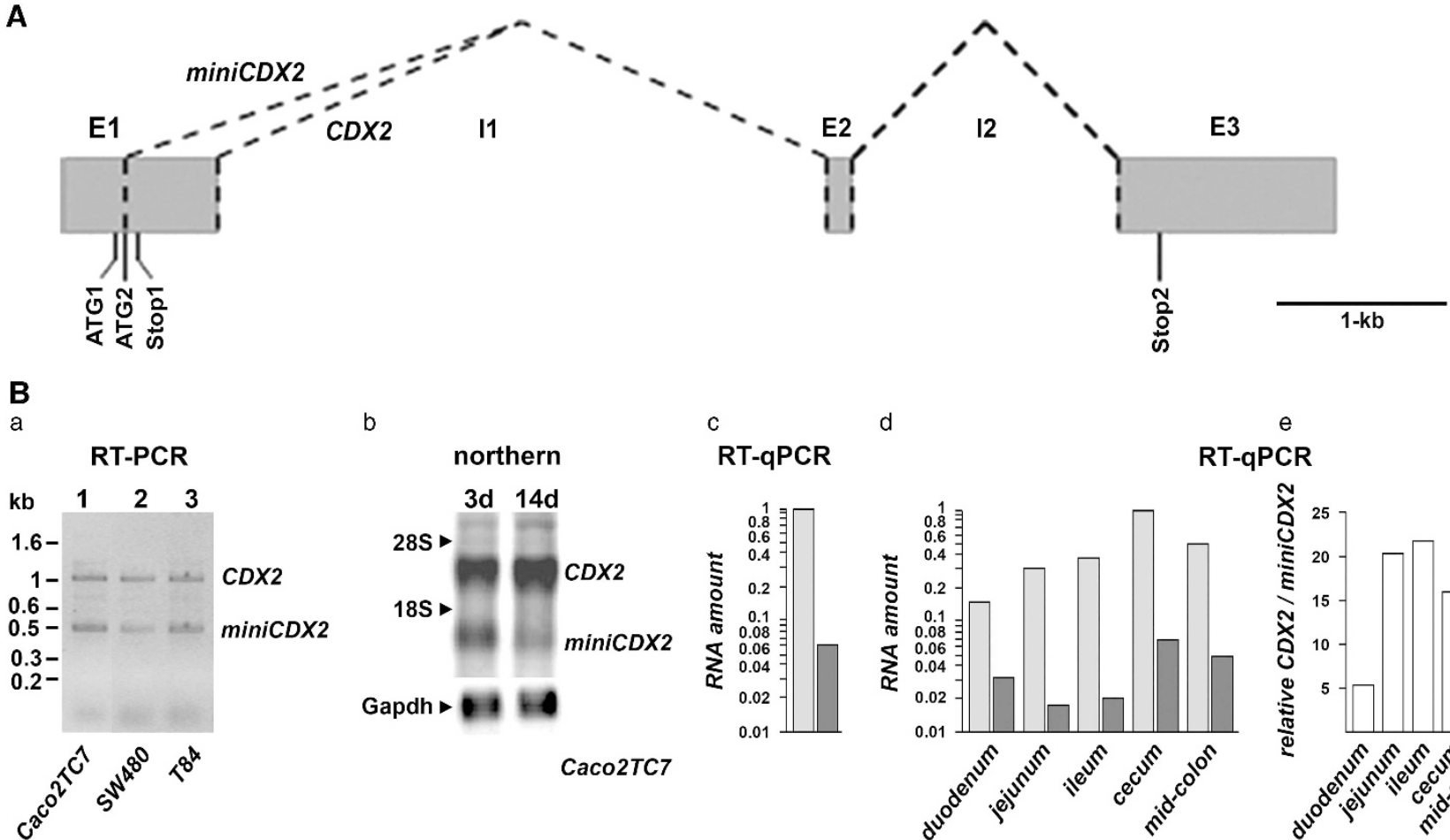

b

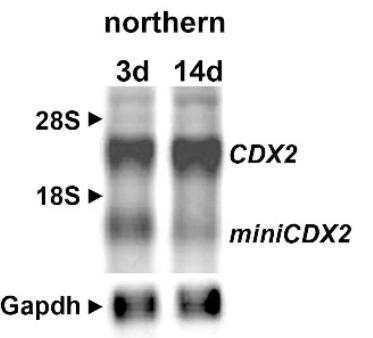

Caco2TC7
$\mathrm{C}$
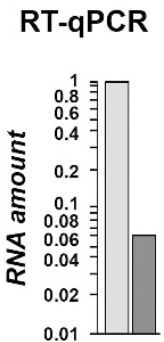

0.01 d

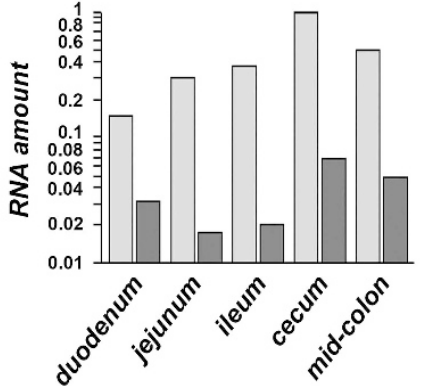

e

CR

Figure 1 Alternative splicing at the CDX2 locus. (A) CDX2 gene map. E1-3: exon-1 to -3; 11 and 2: intron-1 and -2; dotted lines represent the spliced regions to produce, respectively, the CDX2 and miniCDX2 mRNAs. The translation start codons $\mathrm{ATG}_{1}$ and $\mathrm{ATG}_{2}$, and the stop codons $\mathrm{Stop}_{1}$ and $\mathrm{Stop}_{2}$ are indicated. (B) Expression of the miniCDX2 transcript. (a) RT-PCR on intestinal cell lines with the primers CDX21F/CCR hybridizing, respectively, upstream of $A T G_{1}$ in the exon-1 and downstream of Stop 2 in the exon-3. The two bands correspond to the CDX2 and miniCDX2 transcripts. (b) Northern blot of polyA RNA (10 $\mu \mathrm{g}$ per lane) from Caco2TC7 cells cultured for 3 days and 14 days revealing the CDX2 and miniCDX2 mRNAs. (c) RT-qPCR quantification of CDX2 (light gray) and miniCDX2 mRNA (dark gray) in 3 days Caco2TC7 cells (semi-logarithmic scale). (d) RT-qPCR of CDX2 (light gray) and miniCDX2 mRNA (dark gray) along the murine gut (semi-logarithmic scale); CDX2 was arbitrary set at 1 in the cecum. (e) CDX2/ miniCDX2 mRNA ratio along the mouse intestine

miniCDX2 (Figure 1B). The level of this variant was far below that of the CDX2 mRNA (15-times less abundant) in proliferating Caco2TC7 cells ( 3 days in culture) and further decreased in differentiated cells (14 days in culture). The miniCDX2 mRNA was up to 20 -times less abundant than the CDX2 mRNA along the murine intestine.

MiniCX2 corresponds to an evolutionary-conserved alternative splicing variant. The production of the miniCDX2 variant involves a canonical GU splicing donor site within the exon-1, instead of the standard donor site at the end of the exon used to produce the CDX2 mRNA (Supplementary Figure S1). Remarkably, the alternative donor site overlaps the translation initiation codon of the CDX2 protein in the CDX2 mRNA (referred thereafter to as $\mathrm{AUG}_{2}$ ) that is therefore destroyed in the miniCDX2 mRNA. Yet, the CDX2 gene sequence contains one additional AUG located $29 \mathrm{pb}$ upstream of $\mathrm{AUG}_{2}$. This $A \cup G$, referred to as $A \cup G_{1}$, is out-of-frame with $A \cup G_{2}$ but in-frame with the open reading frame of exon-2 and -3 in the miniCDX2 transcript. $A \cup G_{1}$ is preceded by GGCAGC and $A \cup G_{2}$ by GCCACC, both being consistent with a consensus Kozak box (GNCANCAUG). ${ }^{21}$ The CDX2 mRNA, but not the miniCDX2 variant, additionally contains a translation stop codon (Stop1) located four nucleotides downstream of $A \cup G_{2}$, thus in-frame with $A \cup G_{1}$.
Strikingly, the motif composed of (i) the GU splicing donor site overlapping the $A \cup G_{2}$ start codon of the CDX2 protein, (ii) the putative upstream start site $A \cup G_{1}$ and (iii) the stop codon Stop1 in-frame with $A \cup G_{1}$, is evolutionary-conserved in vertebrates from coelacanth to human, including marsupials, birds, reptiles, amphibians, the bichir (the most basal extant ray-finned fish) and the bowfin (the immediate outgroup to teleost fish) (Figure 2A; Supplementary Figure S2). However, it is found neither in $C D X 1$-type genes (except for $C d \times 1 b$ in teleost fishes like Danio rerio, see 'Discussion'), nor in CDX4type genes.

The miniCDX2 variant encodes a truncated form of the CDX2 homeoprotein. To study the translational activity of the upstream $A \cup G_{1}$ and to compare it with $A \cup G_{2}$ used for CDX2 translation, reporter plasmids were constructed in which the $5^{\prime}$-untranslated sequence of the CDX2 gene ending at $A T G_{2}$ (pATG1-ATG2) or ATG 1 (pATG1) was linked to the Luciferase coding sequence (Figure 2Ba). The translation activity of $A \cup G_{2}$ used for CDX2 synthesis was verified by the production of Luciferase in HCT116 cells transfected with pATG1-ATG2 that recapitulates the configuration of the CDX2 mRNA in which $A \cup G_{2}$ is in-frame and $A \cup G_{1}$ out-of-frame with the reporter sequence. Interestingly, pATG1 ending at $A T G_{1}$ and recapitulating the miniCDX2 configuration produced an even higher luciferase activity than pATG1-ATG2. This activity was abolished by mutating ATG 
A

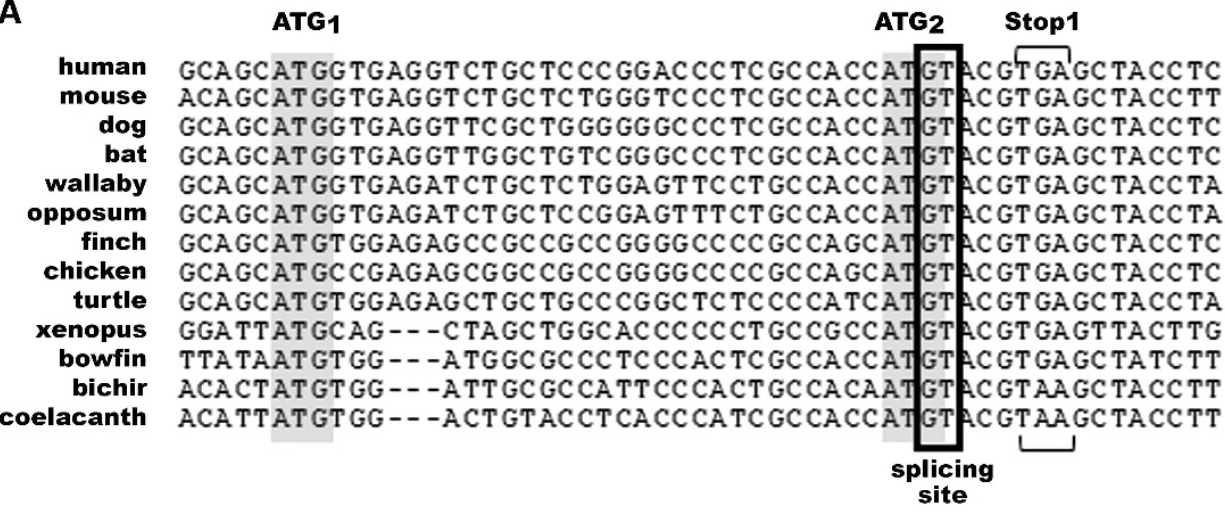

B

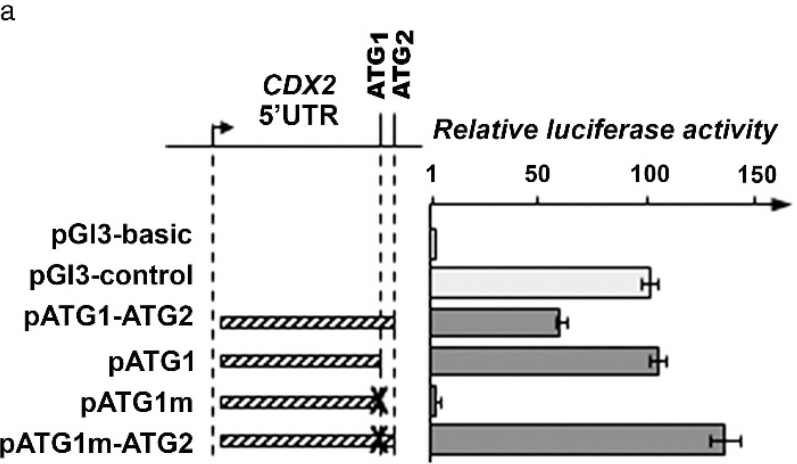

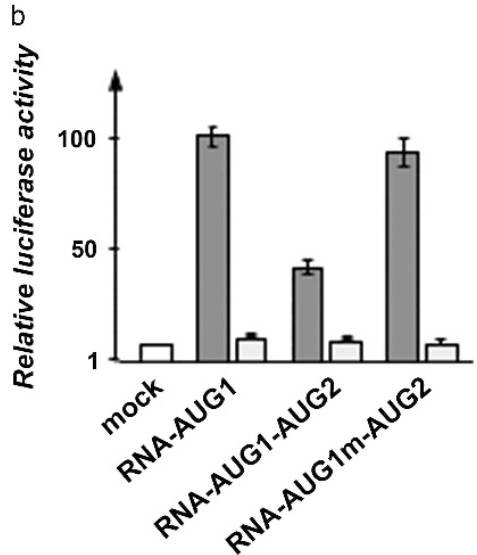

Figure 2 Functionality of the conserved alternative splicing/translation motif of the CDX2 locus. (A) The conserved motif found in CDX2-type genes (see also the Supplementary Figure 2). (B) (a) Luciferase activity in human colon cancer HCT116 cells transfected with the indicated reporter plasmids; $X_{\text {designates the mutation of }}$ ATG $G_{1}$ into TAG in pATG1m and in pATG1m-ATG2; pGI3-control was set to 100 and pGI3-basic gave background activity. (b) Luciferase activity in HCT116 cells transfected with in vitrotranscribed polyadenylated RNA without (light gray) or with $\mathrm{m} 7 \mathrm{G}$ cap (dark gray). Data are given as mean \pm S.D. for triplicates

into TAG in pATG1m, while changing $A T G_{1}$ into $T A G$ in pATG1m-ATG2 increased luciferase compared to pATG1ATG2. Thus, $A \cup G_{1}$ is translationally active and, in the context of the CDX2 mRNA, it partially hinders the translational activity of $A \cup G_{2}$.

In the CDX2 mRNA, the $\mathrm{AUG}_{2}$ start codon used for the CDX2 protein is preceded by the translationally competent $A U G_{1}$. Ribosomes generally start translation at the first AUG after cap-dependent scanning of the 5 '-untranslated region, but can skip it for a second site by leaky scanning ${ }^{22}$ or reach an internal codon using a cap-independent internal ribosome entry site. ${ }^{23}$ To compare the cap-dependency of $A \cup G_{1}$ and $A U G_{2}$, uncapped and $\mathrm{m} 7 \mathrm{G}$-capped reporter RNAs named $R_{N A A_{A 1}}$ and RNA $A_{A \cup G 1-A U G 2}$ were in vitro transcribed from PCR templates derived from pATG1 and PATG1-ATG2, respectively, and transfected in HCT116 cells. Luciferase activity was higher for capped versus uncapped RNA $\mathrm{AUG1}_{\text {, }}$,

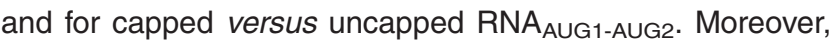
destroying $A_{U} G_{1}$ in $R_{N A} A_{A U G 1 m-A U G 2}$ increased luciferase compared to RNA $A \cup G 1-A \cup G 2$ (Figure 2Bb). Thus, the activity of $A \cup G_{2}$, like $A \cup G_{1}$, is cap-dependent, suggesting that ribosomes reach $A \cup G_{2}$ for $C D X 2$ translation by leaky scanning through $\mathrm{AUG}_{1}$.
The sequence of the splicing mRNA variant predicts the production of a CDX2-related homeoprotein, miniCDX2, in which a 12-aa $N$-terminus segment replaces the 181-aa transactivation domain (Figure 3A, Supplementary Figure S1). An antibody, C2T, was raised against this 12-aa segment and validated using expression plasmids (Figure 3Ba), peptide competition and conditional CDX2 knockout mice (Figure $3 \mathrm{Bc}$ ). Consistent with the presence of the DNAbinding homeodomain, the miniCDX2 protein produced by pFlag-miniCDX2-transfected HCT116 cells was nuclear (Figure 3Bb). Compared to CDX2 present in almost every intestinal crypt and villous epithelial cell from embryos to adults, miniCDX2 was not detected in the embryonic gut, it appeared perinatally at the level of the intervillous epithelium at the origin of the crypts (Figure $3 \mathrm{Ca}$ ), and remained expressed throughout adult life in the small intestinal crypts and in the bottom of the colonic glands (Figures $3 \mathrm{Ca}$ and c). Crypt cells co-expressed miniCDX2 and CDX2 (Figure 3Cb).

In small intestinal tumors of $A p c^{\Delta 14 /+}$ mice, CDX2 expression is known to become reduced and heterogeneous. Interestingly, miniCDX2 was also expressed heterogeneously and in the same tumor regions as CDX2 (Figure 3Da). Moreover, RT-qPCR showed that the ratio between the CDX2 

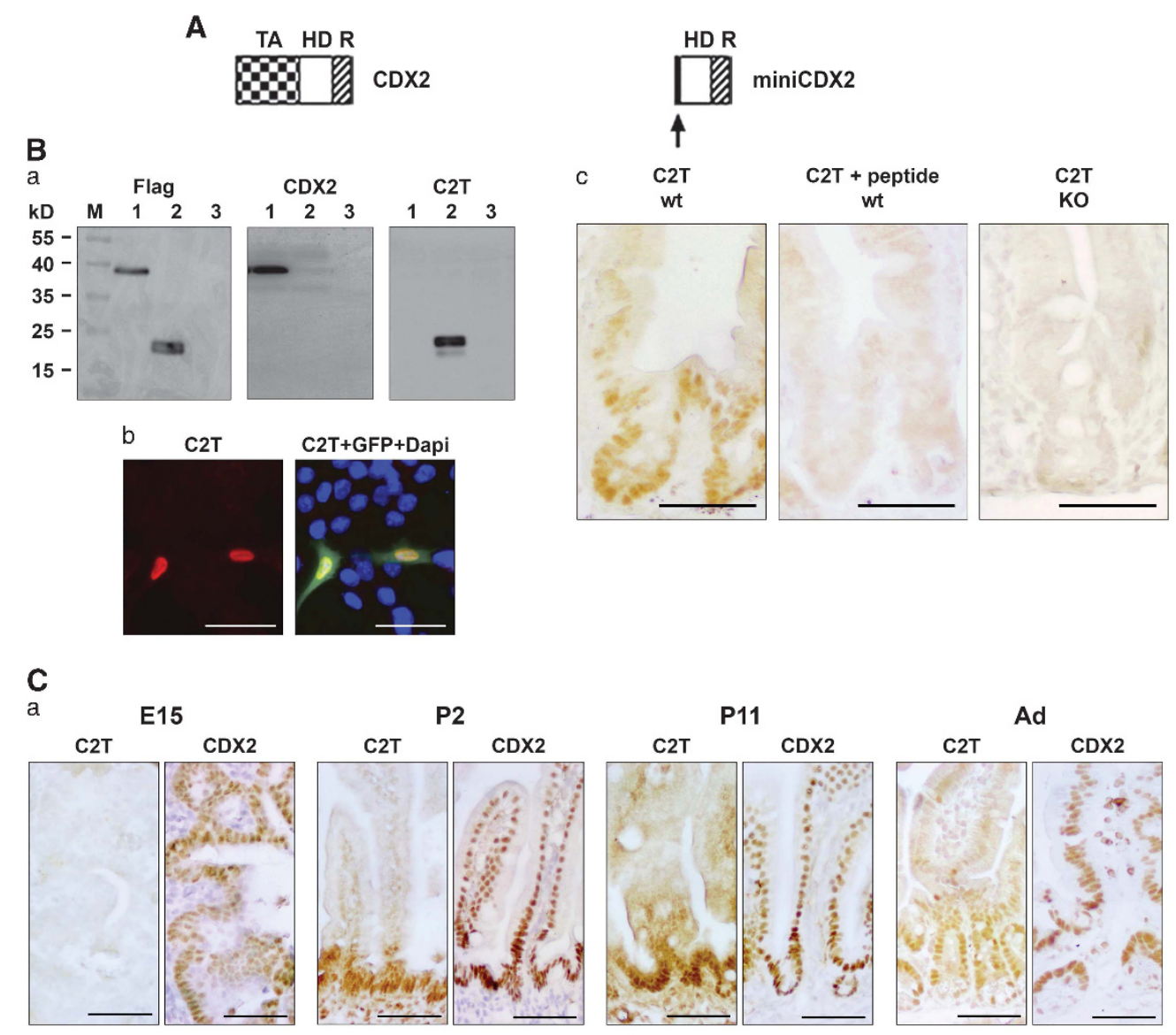

b

CDX2
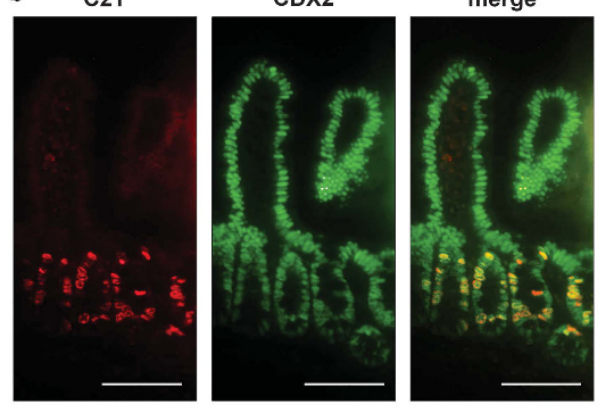

D

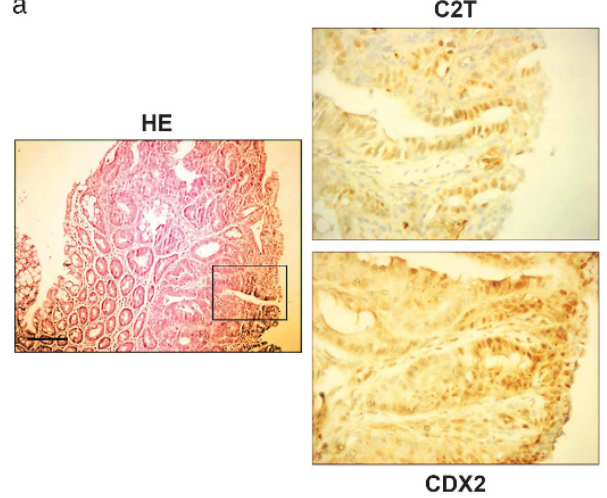

C
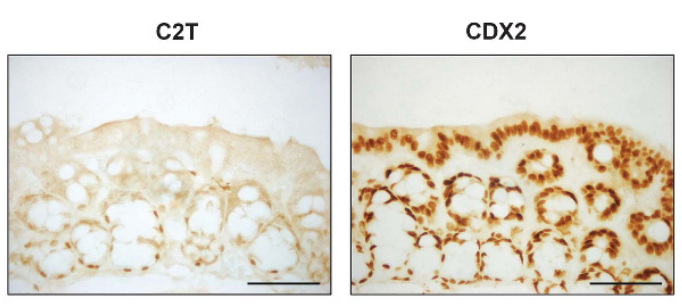

b

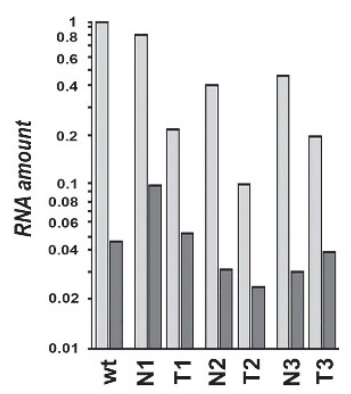

C

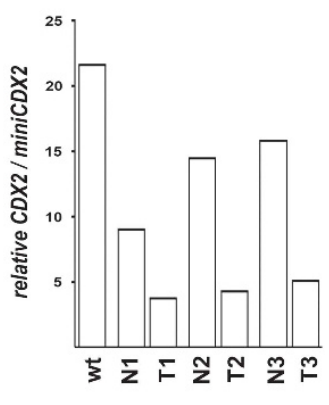


and miniCDX2 transcripts decreased in these tumors compared to the normal mucosa $(P<0.05)$ (Figures 3Db and $c)$.

Opposite and similar molecular functions of miniCDX2 compared to CDX2. We next addressed the molecular functions of miniCDX2 compared to CDX2. As a transcription factor, the CDX2 protein activates a large number of downstream genes including the sucrase-isomaltase (SI) gene. In HCT116 cells transfected with pFlag-CDX2 or pFlagminiCDX2, the CDX2 protein, but not miniCDX2, stimulated the level of SI mRNA and the activity of the SI gene promoter assayed using the $S I$ luciferase reporter; moreover, miniCDX2 dose-dependently abolished the stimulatory effect of CDX2 (Figures 4Aa and b). Chromatin immunoprecipitation (ChIP) revealed the ability of miniCDX2 to bind the same chromatin targets as CDX2, for example, the SI, Li-cadherin and Muc2 promoters (Figure 4Ac), and to reduce the binding of CDX2 to the SI promoter (Figure 4Ad). Thus, miniCDX2 is transcriptionally inefficient, but acts as a dominant-negative regulator of the transcriptional activity of CDX2 by DNAbinding competition.

CDX2 exerts broader functions than regulating transcription through DNA binding. ${ }^{24}$ For instance, it can bind $\beta$-catenin to prevent its interaction with Tcf 4 , thus impeding $\mathrm{Wnt} / \beta$-catenin signaling. ${ }^{25}$ Indeed, CDX2 opposed the stimulation of the Mmp7 mRNA, a Wnt target, and the activity of the TOP-Flash Wnt reporter by $\beta$-catenin/Tcf4 (Figures $4 \mathrm{Ba}$ and $\mathrm{b}$ ). Unlike CDX2, miniCDX2 failed to interfere with the $\beta$-catenin/Tcf4 stimulation of Mmp7 mRNA and TOP-Flash activity. In addition, it failed to relieve the inhibitory effect of CDX2 on $\beta$-catenin/Tcf4 (Figures 4Ba and $\mathrm{b}$ ), and did neither interact with $\beta$-catenin nor prevent $\beta$-catenin/Tcf4 interaction (Figures 4Bc and d). Thus, contrary to its dominant-negative effect on the CDX2 transcriptional activity, miniCDX2 is neutral with respect to the inhibitory function exerted by CDX2 on $\beta$-catenin/Tcf4 signaling.

CDX2 also exerts transcription-independent functions including the increase of $\mathrm{P} 27^{\mathrm{KIP} 1}$ to compromise cell proliferation. ${ }^{26}$ Like CDX2, miniCDX2 increased the level of $\mathrm{P} 27^{\mathrm{KIP} 1}$ protein without changing its mRNA (Figures $4 \mathrm{Ca}$ and $b$ ) and it reduced cell growth in a colony formation assay (Figure 4Cc). The CDX2 protein has also been reported to inhibit double-stranded DNA breaks repair in HCT116 cells by interacting with the KU70/80 complex. ${ }^{27}$ Here, we extended these observations by showing in SW480 colon cancer cells that miniCDX2, like CDX2, also co-immunoprecipitated with KU70/80 proteins, and hampered DNA repair activity (Figures 4Da and b).

Thus, miniCDX2 fine-tunes the activity of CDX2 in that it exerts similar and/or different effects with respect to the multifaceted functions of the full-length homeoprotein: it is dominant-negative on the transcriptional activity of CDX2, it is neutral as regards the inhibitory role of CDX2 on $\beta$-catenin/Tcf4 interaction, and it exerts similar transcriptionindependent effects as CDX2 on cell growth inhibition by $\mathrm{P} 27^{\mathrm{KIP} 1}$ and on double-stranded DNA break repair through KU70/80.

MiniCDX2 participates in the control of intestinal homeostasis. We next explored the role of miniCDX2 in vivo in mice using a gain-of-function approach. For this purpose, transgenic jojo-Flag-miniCDX2 mice were generated, allowing inducible expression of the Flag-miniCDX2 protein (Figure 5A). Crossing jojo-Flag-miniCDX2 and Sox2Cre mice to broadly express Flag-miniCDX2 in the zygote caused embryonic death, which is reminiscent of the lethality resulting from $C D X 2$ knockout (0 living double-transgenics over 80 descendants). In the adult gut of jojo-Flag-miniCDX2 mice (three independent founders), GFP immunostaining used to trace the activity of the CAG promoter revealed only very few villi with transgene expression $(<1 \%$, Supplementary Figure S3Ba). Consequently, jojo-Flag-miniCDX2::VilCre mice generated to target the intestinal epithelium showed only rare villi with Flag-miniCDX2 expression. In those villi with a sustained expression of Flag-miniCDX2, intestinal alkaline phosphatase was lost, whereas gastric Claudin-18 was turned on (Figure 5B), without apparent change in CDX2. This reproduced the differentiation switch induced by targeted $C D X 2$ knockout in the gut, ${ }^{28}$ indicating that miniCDX2 is able to antagonize the transcriptional potential of CDX2 in vivo.

Brunner's glands (BGs) are glandular structures connected to the crypts of the floor of the proximal duodenum. They express both CDX2 and miniCDX2 at low levels (not shown). Interestingly, the CAG promoter is active in the BGs of jojoFlag-miniCDX2 mice, as revealed by GFP immunostaining, thus allowing a widespread expression of Flag-miniCDX2 in the BGs of jojo-Flag-miniCDX2::VilCre mice (Supplementary Figures $\mathrm{S} 3 \mathrm{Bb}$ and $\mathrm{C}$ ). Noteworthy, these mice exhibited a 2.5-fold enlargement of the territory of the BGs (Figure $5 \mathrm{C}$ ).

Figure 3 Protein expression of the miniCDX2 variant. (A) Structure of the CDX2 and miniCDX2 proteins: TA, transactivation domain; HD, DNA-binding homeodomain; R, regulatory domain. The arrow points to the 12-aa Nter peptide of miniCDX2. (B) The C2T antibody raised against the 12-aa Nter peptide of miniCDX2. (a) HCT116 cells transfected with the plasmid pFlag-CDX2 (lane 1), pFlag-miniCDX2 (lane 2) or the empty vector pFlag-CMV2 (lane 3) were analyzed by western blot with Flag, CDX2 or C2T antibodies. The Flag antibody reveals both Flag-CDX2 and Flag-miniCDX2 proteins, whereas the CDX2 and C2Tantibodies, respectively, detect Flag-CDX2 and Flag-miniCDX2. (b) HCT116 cells co-transfected with pFlag-miniCDX2 and pEGFP showed nuclear localization of Flag-miniCDX2 (Flag antibody, red). Bars: $10 \mu$ m. (c) Validation of the C2T antibody in mouse intestinal sections: nuclear immunostaining in the crypt epithelium of wild-type mice; loss of staining when C2T is preincubated with an excess of immunogenic peptide; absence of immunostaining in the intestinal epithelium of conditional knockout (KO) $C d x 2^{f / f}: . A h C r e^{E R T}$ mice. ${ }^{7}$ Bars: $50 \mu$ m. (C) Immunostaining pattern of the miniCDX2 (C2T antibody) and CDX2 proteins in the small intestine of E15 mouse embryos, and of post-natal day 2 (P2) and day 11 (P11) suckling and adult (Ad) mice. (b) Coimmunofluorescence detection of the miniCDX2 (C2T) and CDX2 proteins in the small intestine of adult mice. Bars: $100 \mu \mathrm{m}$. (c) Immunostaining of the miniCDX2 (C2T) and CDX2 proteins in the proximal colon of adult mice. Bars: $50 \mu \mathrm{m}$. Higher magnification pictures are given in the Supplementary Figure S3A. (D) MiniCDX2 expression in tumors of the proximal ileum of $A p c^{\Delta 14 /+}$ mice. (a) HE staining and C2T and CDX2 immunostaining. The region boxed in HE is shown in the immunohistochemistry pictures. Bars: $100 \mu$ m. (b) RT-qPCR quantification of CDX2 (light gray) and miniCDX2 mRNA (dark gray) (semi-logarithmic scale) in wild-type ileum (wt), in three ileal tumors of $A p c^{\Delta 14 /+}$ mice (Apc T1-3) and in the adjacent normal mucosa (Apc N1-3); data are related to the amount of CDX2 mRNA arbitrary set at 1 in the wild-type ileum. (c) Ratio between the CDX2 and miniCDX2 transcripts 
This was accompanied by a higher expression of $P D X 1$, an important regulator of BG morphogenesis ${ }^{29}$ (Figure 5D) without change in cell proliferation and differentiation, as assessed by the absence of significant phospho-histone-3 labeling, and by the staining of neutral mucins (Figure 5E).
To address the consequence of the overgrowth of the BGs, mice were left to age until 18-24 months $(n=19)$. Fifty-two percent (10/19) of jojo-Flag-miniCDX2::VilCre mice developed one to two polyps in the pyloric region (Figure $6 \mathrm{~A}$ ), in contrast to control jojo-Flag-miniCDX2 animals (1/12). These polyps
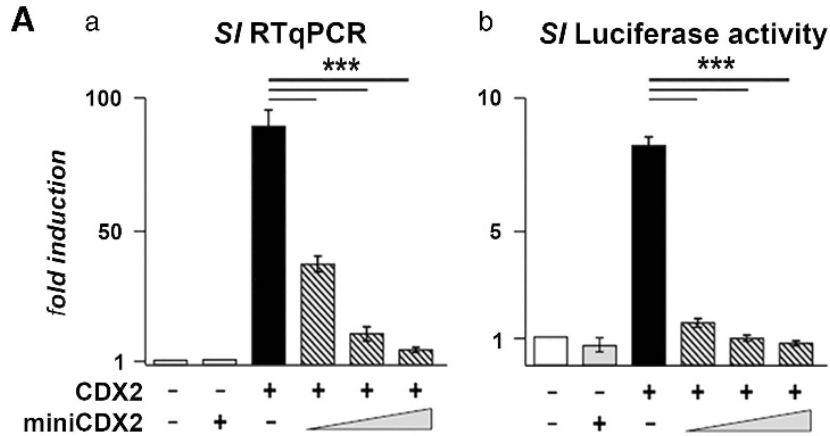

B a

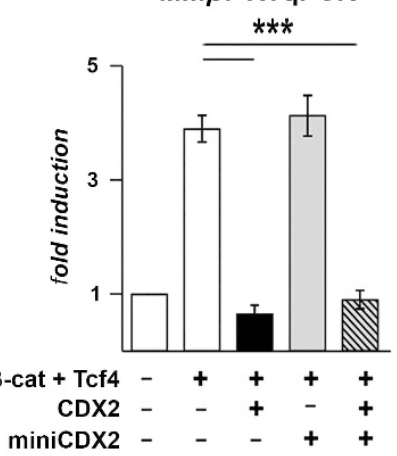

C a

P27 Western blot

b TOP-Flash activity

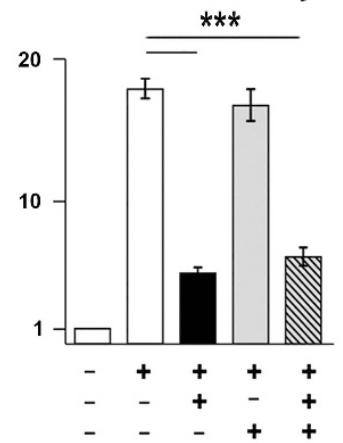

b P27 RTqPCR
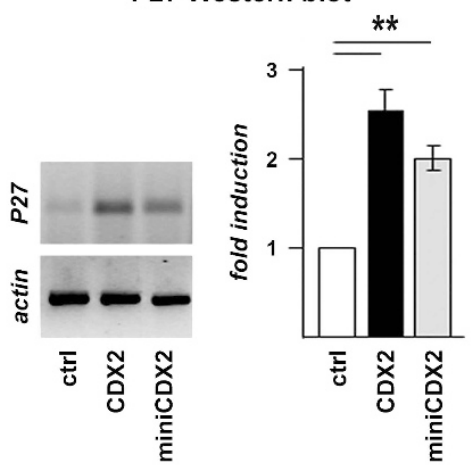

C Clonogenic assay
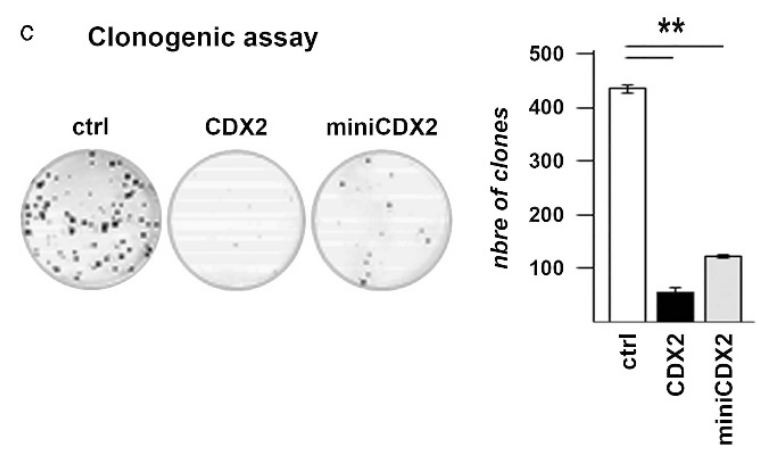

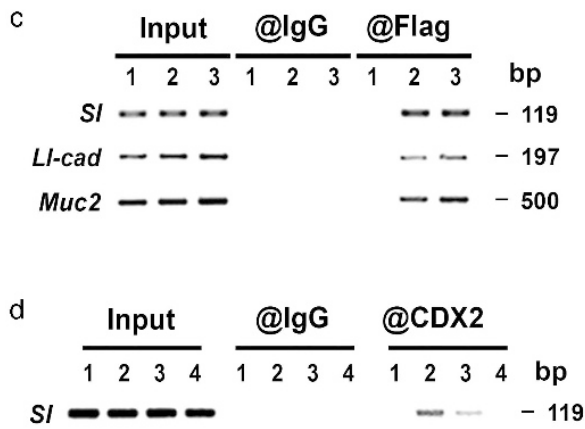

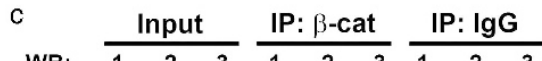
WB: $1 \begin{array}{llllllll}1 & 2 & 3 & 2 & 3 & 1 & 2 & 3\end{array}$ $\beta$-cat $-\infty-100$ $\begin{aligned} \stackrel{ }{\text { Flag }}- & -40 \\ & -35\end{aligned}$

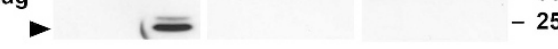

d WB: $\frac{\text { IP: IgG }}{1}$\begin{tabular}{llllll} 
IP: $\beta$-cat \\
\hline 2 & 3 & 4 & 5 & 6
\end{tabular}

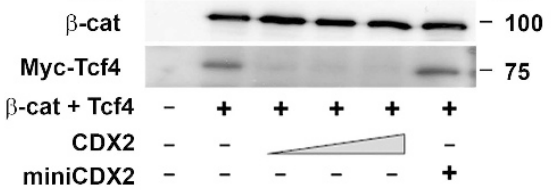

D

a

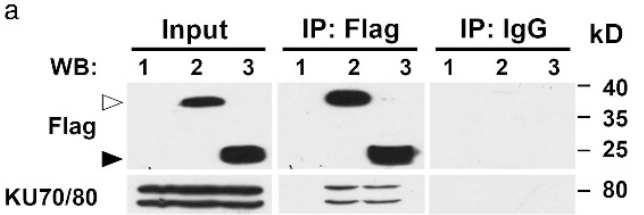

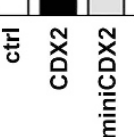

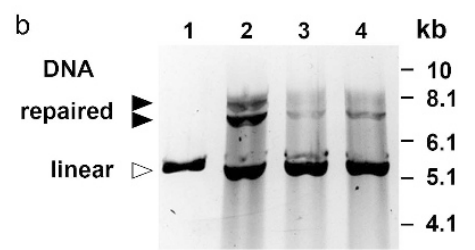


exhibited low- to high-grade dysplasia, without sign of neoplasia. As illustrated in Figures $6 \mathrm{~A}$ and $\mathrm{B}$, they were formed by expanded BGs extending even under the gastric mucosa, covered by disorganized crypts from the overlying duodenal epithelium, and by a surface epithelium with foreguttype properties, exhibiting gastric Claudin-18 expression and occasionally P63-positive squamous stratified epithelium. Phospho-histone-3 proliferating cells concentrated in the disorganized crypt area. Consistent with the absence of neoplastic area, there was no evidence of $\beta$-catenin cytoplasmic/nuclear translocation. BGs produce EGF while adenomas develop after deletion of the EGF-receptor inhibitor Lrig1. ${ }^{30}$ RT-qPCR revealed a 4-18 times higher level of EGF mRNA in the polyps of jojo-Flag-miniCDX2::VilCre mice compared to the duodenal mucosa of control animals $(n=3)$. Linked to that, the disorganized crypt area showed elevated levels of
A

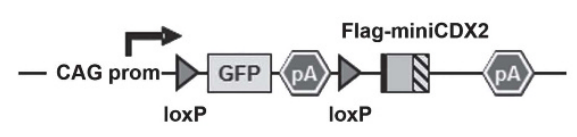

B
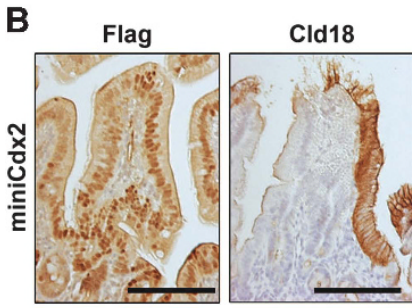

ctrl
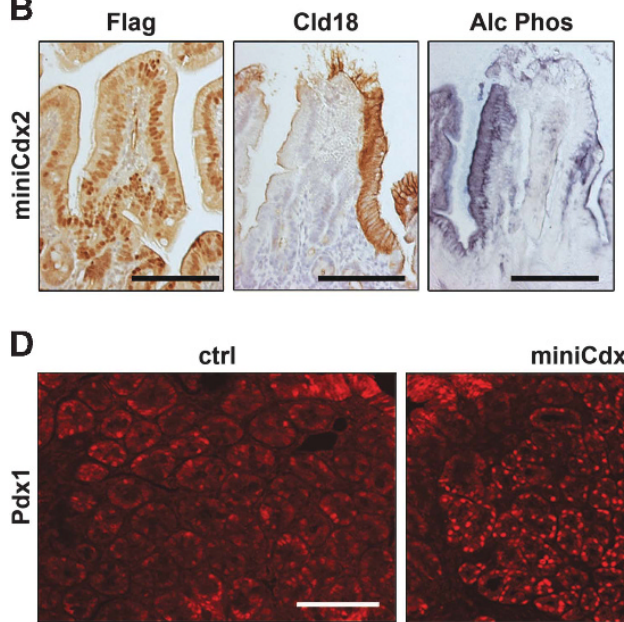

C

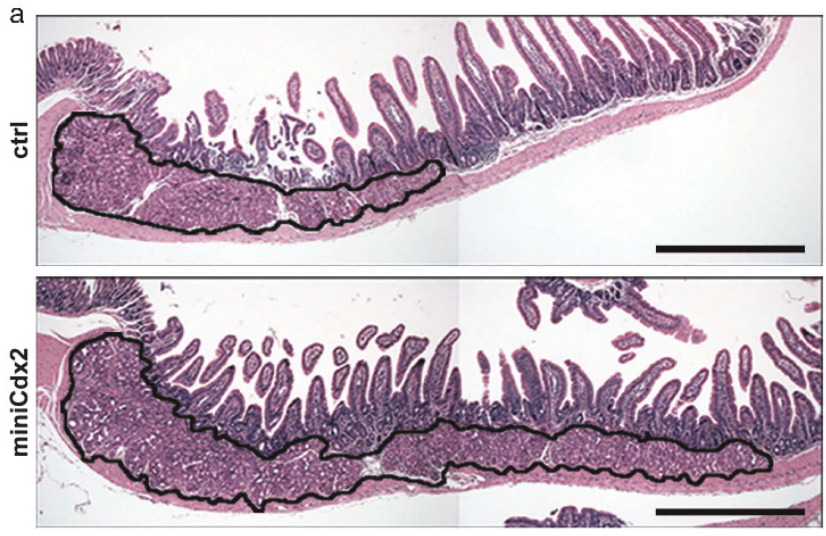

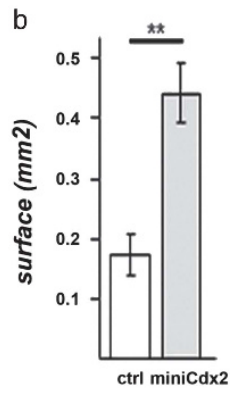

E
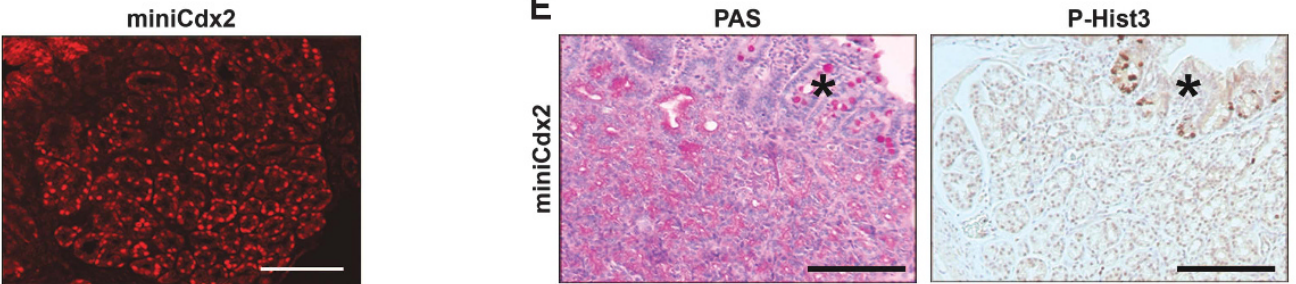

Figure 5 Overexpression of miniCDX2 in transgenic mice. (A) Map of the jojo-Flag-miniCDX2 transgene with the CAG promoter, the loxP/Cre-excisable cassette containing the GFP coding sequence followed by a transcriptional stop (pA), and the Flag-miniCDX2 coding sequence. (B) Serial sections of rare small intestinal villi of jojo-Flag-miniCDX2:: VilCre mice showing Flag-miniCDX2 expression accompanied by the loss of alkaline phosphatase and the ectopic expression of Claudin-18. Bars: $50 \mu \mathrm{m}$. (C) (a) Histology of the Brunner's glands of jojo-Flag-miniCDX2::VilCre mice (miniCdx2) compared to control jojo-Flag-miniCDX2 animals (ctr); bars: $500 \mu$ m. (b) Quantification of the surface of the Brunner's glands in 3-4-month-old control jojo-Flag-miniCDX2 (ctrl, $n=12$ ) and jojo-Flag-miniCDX2::VilCre mice (miniCdx2, $n=9$ ); ${ }^{* *} P<0.01$. (D) Pdx1 immunofluorescence staining in control jojo-Flag-miniCDX2 (ctrl) and jojo-Flag-miniCDX2::VilCre (miniCdx2) mice; bars: $100 \mu \mathrm{m}$. (E) The Brunner's glands of the jojo-Flag-miniCDX2::VilCre mice show no major change in cell differentiation (PAS staining) and no obvious activation of the cell proliferation (P-Hist3). Bars: $100 \mu \mathrm{m}$. The asterisks label the duodenal crypts overlaying the Brunner's glands

Figure 4 Molecular functions of miniCDX2 compared to CDX2. (A) Effect of miniCDX2 on the transcriptional activity of the CDX2 protein. (a) RT-qPCR quantification of the SI RNA and (b) pSI-luc luciferase activity in HCT116 cells transfected either with pFlag-miniCDX2 or pFlag-CDX2 or pFlag-CDX2 together with increasing amounts of pFlagminiCDX2; data are given as mean \pm S.D. for triplicates $\left.{ }^{* * *} P<0.001\right)$. (c) ChIP with anti-Flag or lgG in HCT116 cells transfected with pFlag-CMV2 (lane 1), pFlag-CDX2 (lane 2) or pFlag-miniCDX2 (lane 3); co-precipitated DNA was PCR-amplified with primers of the SI, LI-cadherin and Muc2 promoters. (d) ChIP with anti-CDX2 or IgG in HCT116 cells transfected either with pFlag-CMV2 (lane 1) or pFlag-CDX2 (lane 2) or pFlag-CDX2 together with a threefold molar excess of pFlag-miniCDX2 (lane 3) or pFlag-miniCDX2 (lane 4); co-precipitated DNA was PCR-amplified with primers of the S/ promoter. (B) Effect on miniCDX2 on $\beta$-catenin/Tcf4 signaling. (a) RT-qPCR quantification of the Mmp7RNA and (b) TOP-Flash luciferase activity in HEK293 cells transfected with pS33A- $\beta$-catenin, pMyc-Tcf4, pFlag-CDX2 and/or pFlag-miniCDX2; data are given as mean \pm S.D. for triplicates $\left.{ }^{* \star \star} P<0.001\right)$. (c) Proteins from cells transfected either with $p S 33 A-\beta$-catenin alone (lane 1), or pS33A- $\beta$-catenin together with pFlag-CDX2 (lane 2 ), or pS33A- $\beta$ catenin together with pFlag-miniCDX2 (lane 3) were immunoprecipitated with anti- $\beta$-catenin or IgG, and analyzed by western blot with anti- $\beta$-catenin and with anti-Flag to reveal Flag-CDX2 $(\triangleright)$ and Flag-miniCDX2 $(\triangleright)$. (d) Proteins from cells transfected with pS33A- $\beta$-catenin and pMyc-Tcf4 together with increasing amounts of pFlag-CDX2 or pFlagminiCDX2 were immunoprecipitated with anti- $\beta$-catenin, and analyzed by western blot with anti- $\beta$-catenin and anti-Myc to check the $\beta$-catenin/Tcf4 interaction. (C) Effect of miniCDX2 on P27 $7^{\mathrm{KIP} 1}$. (a) Representative western blot and quantification and (b) RT-qPCR quantification of P2 $7^{\mathrm{KIP} 1}$ protein and RNA in HCT116 cells transfected either with pFlag-CMV2 (ctrl) or pFlag-CDX2 or pFlag-miniCDX2. (c) Representative pictures and quantification of clonogenic assays on HCT116 cells transfected either with pFlag-CMV2 (ctrl) or pFlag-CDX2 or pFlag-miniCDX2. Data are means \pm S.D. for triplicates $\left({ }^{* *} P<0.01\right)$. (D) Effect of miniCDX2 on double-strand break DNA repair. (a) Proteins from cells transfected either with pFlag-CMV2 (lane 1), or pFlag-CDX2 (lane 2) or pFlag-miniCDX2 (lane 3) were immunoprecipitated with anti-Flag or lgG, and analyzed by western blot with anti-Flag and anti-KU70/80 to check the interaction of Flag-CDX2 ( $\triangleright$ ) or Flag-miniCDX2 $(-)$ with the KU70/80 complex. (b) For DNA repair assays, linearized plasmid pcDNA3 (lane 1) was incubated with nuclear extracts of SW480 cells transfected with pFlag-CMV2 (lane 2), pFlag-CDX2 (lane 3) or pFlag-miniCDX2 (lane 4), and then separated by electrophoresis on an agarose gel. The linear and repaired (circular and/or concatemers) plasmid forms are indicated 
A a

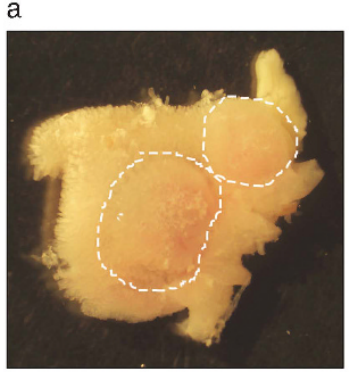

B
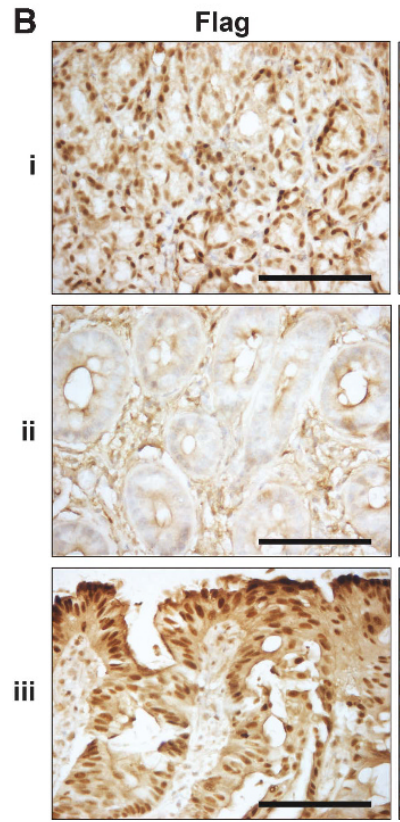

b
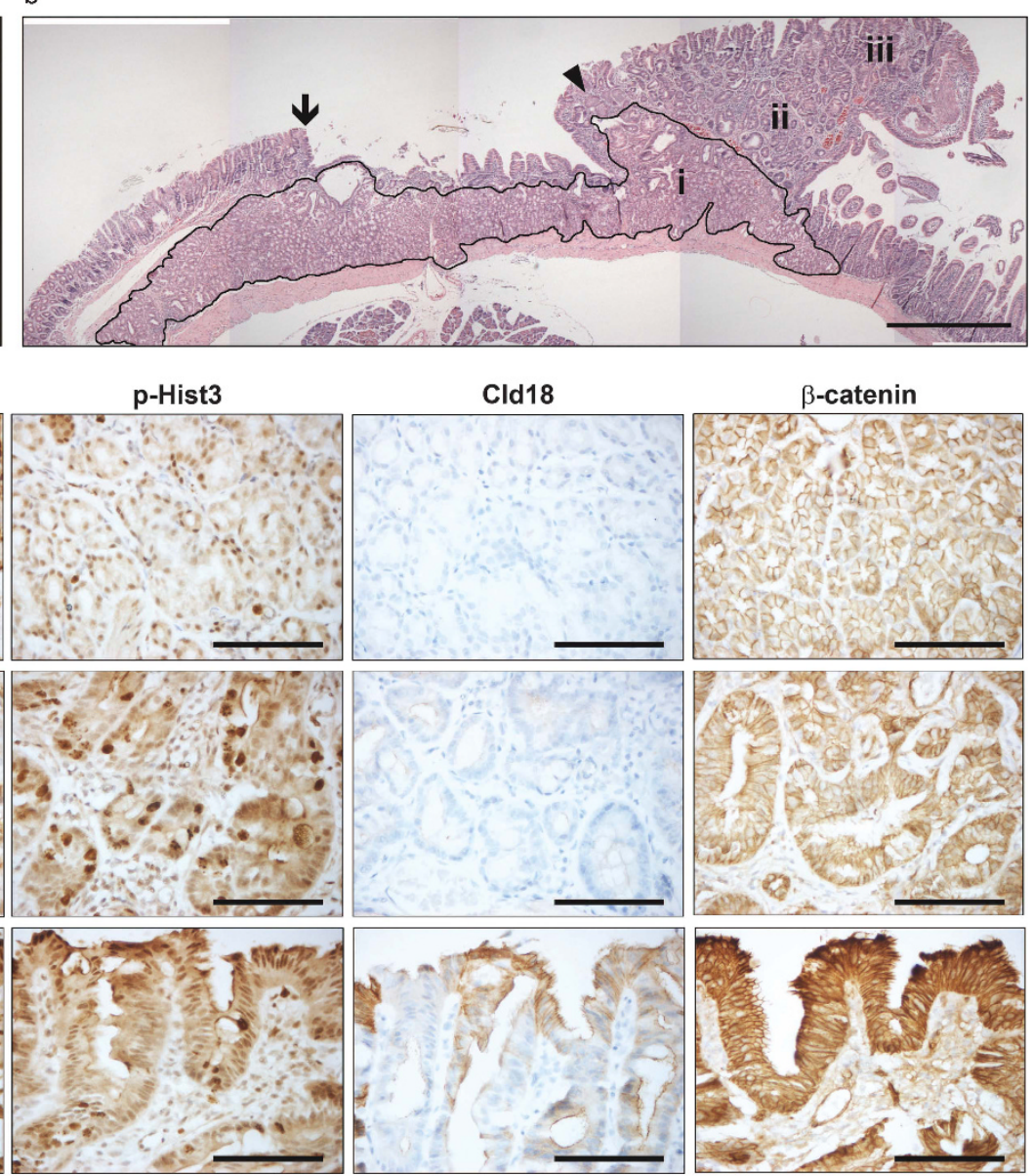

C

i
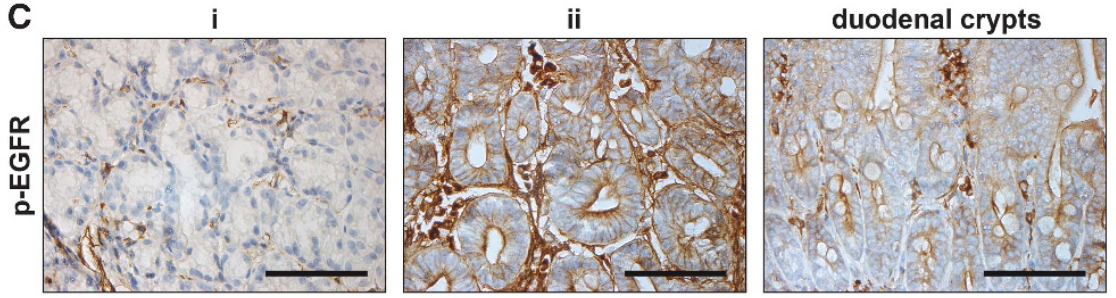

Figure 6 Duodenal lesions in aged jojo-Flag-miniCDX2::VilCre mice. (A) (a) Macroscopic view of two adjacent polyps grown in the duodenal region of a 18-month-old jojoFlag-miniCDX2::VilCre mouse. (b) Histology of a polyp near the gastric-intestinal boundary (arrow); (i), (ii) and (iii), respectively, designate the expanded territory of Brunner's glands (encircled), the hyper-proliferating crypts and the surface area. The arrowhead locates squamous stratified epithelium. Bar: $500 \mu \mathrm{m}$. (B) Immunostaining of FlagminiCDX2, phospho-histone-3, Claudin-18 and $\beta$-catenin in the regions (i), (ii) and (iii) of the duodenal polyp. Bars: $50 \mu \mathrm{m}$. (C) Phospho-EGFR immunodetection in the regions (i) and (ii) of the polyps, and in normal adjacent duodenal crypts. Bars: $50 \mu \mathrm{m}$

membranous phospho-EGF-receptor, as compared to normal adjacent duodenal crypts (Figure 6C). Thus, the miniCDX2driven expansion of BGs leads to the overproduction of EGF that activates the EGFR and makes hyperproliferate the overlaying duodenal crypts, ultimately forming polyps.

Autoregulation of the CDX2 pre-mRNA splicing. $C d \times 2^{+/-}$ mice develop cecal heteroplasias exhibiting a shift toward a foregut-type differentiation. ${ }^{3}$ These lesions are devoid of CDX2 protein even though one gene copy is preserved. Since miniCDX2 overexpression also induces foregut-type transdifferentiation, this prompted us to look for miniCDX2 in the heteroplasias of $\mathrm{Cdx2^{+/- }}$ mice. Unlike CDX2 present in the normal cecal epithelium but absent in the heteroplastic glands, miniCDX2 was detected not only in the normal epithelium but also in the heteroplastic structures, indicating a splicing shift in favor of miniCDX2 in the heteroplasias (Figure 7a). This opens the possibility that the balance between both splicing forms might be regulated and depend on the CDX2 protein itself.

To investigate the mechanism of pre-mRNA splicing, we constructed the plasmid eC2I1 overlapping the exon-1, intron1 and the fused exon-2/-3 of the CDX2 gene, and having a Flag sequence preceding the Stop codon in exon-3 (Figure 7b). In HCT116 cells, eC2l1 encoded both CDX2Flag and miniCDX2-Flag variants identified using Flag, CDX2 


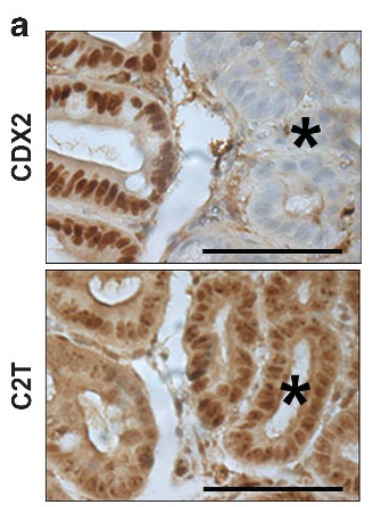

b
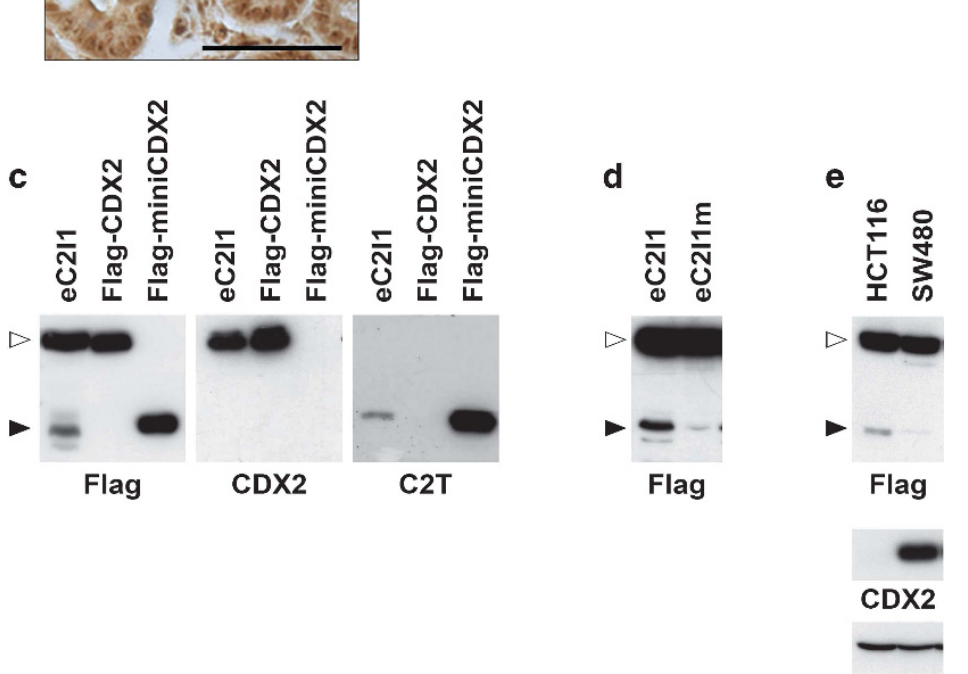

Actin

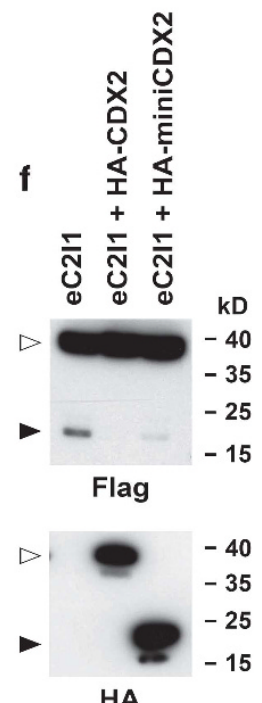

Figure 7 Autoregulation of the CDX2 pre-mRNA splicing. (a) Immunodetection of the CDX2 and miniCDX2 (C2T antibody) proteins at the border between normal cecal epithelium and heteroplasia (asterisk) in $C d x 2^{+/-}$mice. Bars: $50 \mu \mathrm{m}$. (b) Map of the eC211 reporter plasmid. (c) Production of the two protein variants CDX2-Flag ( $\triangleright$ ) and miniCDX2-Flag $(-)$ from the reporter plasmid eC211. HCT116 cells were transfected either with the plasmids eC211, pFlag-CDX2 or pFlag-miniCDX2, and the proteins were revealed using anti-Flag, anti-CDX2 and anti-miniCDX2 (C2T) antibodies, respectively. (d) HCT116 cells transfected with the plasmid eC211 or with the mutant form eC211m. (e) Inverse correlation between the production of miniCDX2-Flag and the endogenous level of CDX2 in HCT116 and SW480 colon cancer cell lines. Upper panel: HCT116 and SW480 transfected with eC211. Lower panel: endogenous level of expression of CDX2 protein in HCT116 and SW480 cells related to actin. (f) HCT116 cells transfected with eC211 and either the control empty plasmid, or the plasmids encoding pHA-CDX2 or pHA-miniCDX2. The lower panel confirms the expression of HA-CDX2 and HA-miniCDX2 in these cells, using anti-HA antibody

and C2T antibodies (Figure 7c). Mutating the GT alternative splicing donor site into GA to give eC211m compromised the production of miniCDX2-Flag (Figure 7d). Thus, eC211 represents an appropriate reporter system of the alternative splicing of the CDX2 pre-mRNA.

Interestingly, transfecting eC2l1 in HCT116 and SW480 cells produced different rates of miniCDX2-Flag protein, inversely related to the respective endogenous level of CDX2 in these cells (Figure 7e). To address a possible role of the CDX2 protein in regulating its pre-mRNA splicing, HCT116 cells were co-transfected with eC2I 1 and the plasmid encoding HA-CDX2. HA-CDX2 reduced the amount of miniCDX2-Flag produced by eC2l1, and this effect was also recapitulated by the transcription-deficient protein HAminiCDX2 (Figure 7f). Thus, the CDX2 and miniCDX2 proteins exert a similar inhibitory effect on the alternative splicing leading to the miniCDX2 variant, through a transcriptionindependent mechanism.

The sequence around the alternative splicing donor site of the CDX2 pre-mRNA revealed several putative splicing factors binding elements conserved in human and mouse (Supplementary Figure 4), in particular for the SRp30c and ASF/SF2 factors expressed in intestinal cell lines (Figure 8A). In transfected HCT116 cells, His-ASF/SF2 inhibited the splicing leading to miniCDX2-Flag, whereas His-SRp30c stimulated it; in addition, the stimulatory effect exerted by His-SRp30c was abolished by His-ASF/SF2 (Figure 8Ba). Of note, CDX2 also counteracted the effect of SRp30c (Figure 8Bb). Co-immunoprecipitation (colP) was performed to investigate possible interactions between these factors. In transfected HCT116 cells, His-SRp30c co-immunoprecipitated with His-ASF/SF2 (Figure $8 \mathrm{Ca}$ ). A similar result was obtained with the endogenous SRp30c and ASF/SF2 proteins, and the amount of co-precipitated material increased by RNase treatment, indicating an RNA-independent interaction (Figure $8 \mathrm{Cb}$ ). Interestingly, HA-CDX2 also interacted with the endogenous ASF/SF2 (Figure 8Da) and SRp30c proteins (Figure 8Ea), and the amount of complex increased by RNase treatment. HA-miniCDX2 gave the same results, showing that the transactivation domain and activity of CDX2 is dispensable 
A

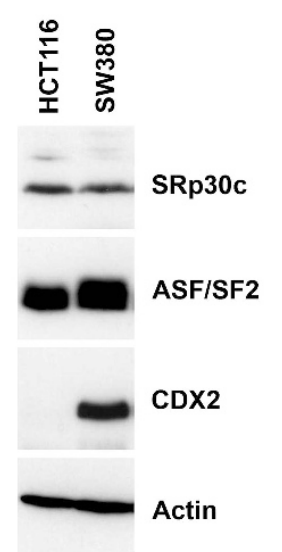

C

a

@Actin

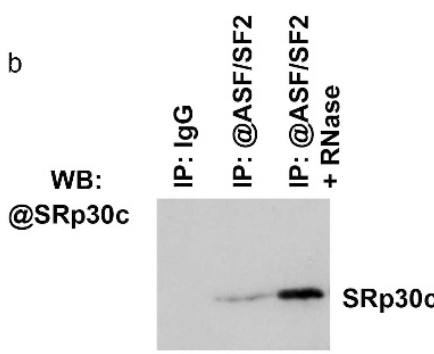

F a

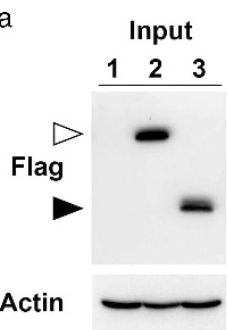

B

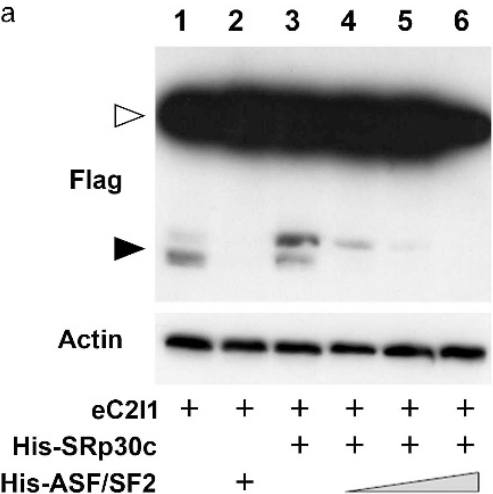

D

a Input IP: @HA

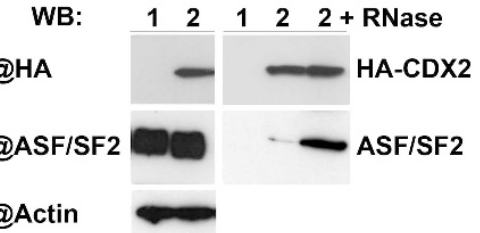

b

WB: Input IP: @HA

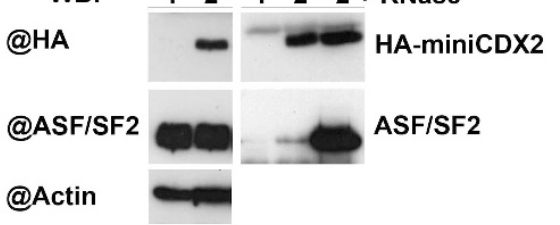

IP: @ASF/SF2

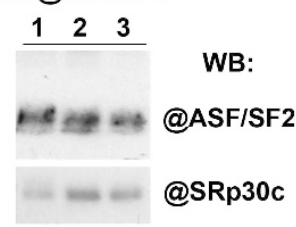
b b

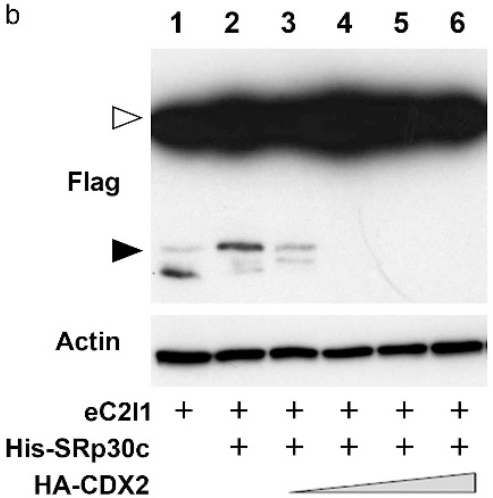

E

a Input IP: @HA

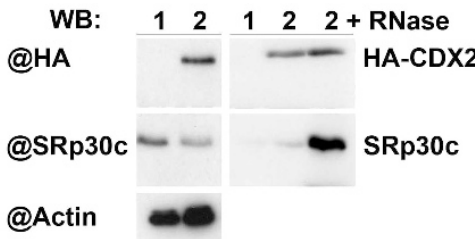

b Input IP: @HA

WB: $\begin{array}{lllll}1 & 2 & 1 & 2 & 2 \text { + RNase }\end{array}$ @HA - HA-miniCDX2 @SRp30c $\ldots$ SRp30c @Actin

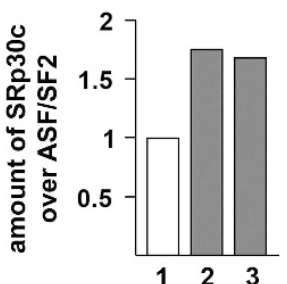

Figure 8 Mechanism of regulation of the CDX2 pre-mRNA splicing. (A) Expression of the splicing factors ASF/SF2 and SRp30c in HCT116 and SW480 colon cancer cells. (B) Impact of ASF/SF2, SRp30c and CDX2 on the reporter plasmid eC211. HCT116 cells were transfected with eC2I1 and the expression plasmids for the indicated proteins, and the two splicing variants CDX2-Flag $(\triangleright)$ and miniCDX2-Flag $(\rightarrow)$ were detected with anti-Flag antibody. (C) Interaction between ASF/SF2 and SRp30c. (a) HCT116 cells were transfected with the plasmids encoding His-ASF/SF2 and His-SRp30c. After immunoprecipitation with anti-ASF/SF2 antibody or with IgG, the proteins were revealed with antiASF/SF2 and anti-SRp30c antibodies. (b) Endogenous proteins of HCT116 cells were immunoprecipitated with lgG or with anti-ASF/SF2 antibody without or with pre-treatment with RNase, and revealed with anti-SRp30c antibody. (D) Interaction between ASF/SF2 and CDX2 or miniCX2. (a) HCT116 cells were transfected with the control plasmids pCMV2-Flag (lane 1) or pHA-CDX2 (lane 2). Protein extracts immunoprecipitated with anti-HA beads, and revealed with anti-HA and anti-ASF/SF2 antibodies. (b) Same as (a) with pHA-miniCDX2 instead of pHA-CDX2. (E) Interaction between SRp30c and CDX2 or miniCDX2. Same as (D) except that protein detection used anti-SRp30c instead of antiASF2/SF2 antibody. (F) Effect of CDX2 and miniCDX2 on the interaction between ASF/SF2 and SRp30c. (a) HCT116 cells were transfected with the control plasmid pCMV2-Flag (lane 1), with pHA-CDX2 (lane 2) or pHA-miniCDX2 (lane 3). Cell extracts were immunoprecipitated with anti-ASF/SF2 antibody, and revealed with anti-ASF/SF2 and anti-SRp30c antibodies. (b) Quantification of immunoprecipitated SRp30c relative to ASF/SF2 
for its interaction with splicing factors (Figures 8Db). Hence, we investigated the impact of CDX2 and miniCDX2 on the ASF/SF2 / SRp30c complex. For this purpose, cells were transfected either with the control plasmid or pHA-CDX2 or pHA-miniCDX2, and nuclear extracts were processed for immunoprecipitation with anti-ASF/SF2 antibody. As shown in Figure 8F, the amount of SRp39c co-immunoprecipitated with ASF/SF2 increased in the presence of HA-CDX2 or HA-miniCDX2, respectively, by 1.7- and 1.6-fold compared to the control. Thus, CDX2 and miniCDX2 stimulate the SRp30c/ ASF/SF2 interaction, which subsequently hampers the selection of the alternative splicing donor site used to produce the miniCDX2 splicing variant.

\section{Discussion}

This work reports a number of novel exon-exon junctions for genes expressed in the gut, which deserves future studies of the relevance of RNA splicing in intestinal development, homeostasis and disease. Focusing on the homeobox gene $C D X 2$, a major regulator of gut ontogenesis and function, $4,8,28$ we identified the miniCDX2 variant, resulting from the combination of alternative pre-mRNA splicing and alternative translation initiation. This variant fine-tunes CDX2 activity through versatile functions regarding the full-length homeoprotein: miniCDX2 antagonizes the transcriptional activity of CDX2 by DNA-binding competition, it is neutral with respect to the inhibitory function exerted by CDX2 on $\beta$-catenin/Tcf 4 signaling, and it has the same activities as CDX2 on cell proliferation, DNA repair and RNA splicing through P27, KU70/80 and splicing factors, respectively. The production of miniCDX2 is based on a conserved sequence motif among $C D X 2$ paralog in vertebrates, that is absent in other members of the caudal family, with the exception of $C d \times 1 b$ in teleost fishes. Interestingly, in teleosts, the CDX2-type gene has been lost and replaced by a duplicated version of $C D X 1(C d x 1 b$ in $D$. rerio), which is functionally equivalent to the mammalian CDX2 gene for intestinal development. ${ }^{31}$ Thus, $C d x 1 b$, unlike $C d x 1 a$, may have acquired a similar motif as $C D X 2$, although being a gene of the CDX1 type. The conserved motif of the $C D X 2$-type genes has implications for translation initiation. Indeed, in the context of the CDX2 mRNA, the $\mathrm{AUG}_{2}$ codon used for CDX2 translation is preceded by the upstream and out-of-frame $\mathrm{AUG}_{1}$ that could potentially initiate the synthesis of a 13-aa peptide. Although the existence of this peptide remains elusive, short upstream open reading frames are relatively frequent and can control the translation of the downstream open reading frame or even be involved in diseases. $^{32}$

In the gut, both the loss or the excessive expression of CDX2 are lethal, ${ }^{8,9}$ highlighting the need for a precise regulation of the activity of the gene. Previous studies have already reported transcriptional ${ }^{33,34}$ and post-translational levels of regulation. ${ }^{35,36}$ This study adds a novel posttranscriptional level of regulation through the production of the variant miniCDX2 that fine-tunes the activity of CDX2. Another variant of CDX2 has been reported, CDX2-A/S, described as a regulator of splicing. ${ }^{37}$ The present study further uncovers that the transcription factor CDX2 and its variant miniCDX2 also act as splicing regulators through a non-transcriptional mechanism involving their interaction with splicing factors. This involvement in pre-mRNA splicing might considerably broaden the sphere of activity of the CDX2 homeobox gene. Interestingly, miniCDX2 is expressed in the crypts, like the S60-phosphorylated form of CDX2 characterized by a reduced transcriptional activity. ${ }^{36}$ This suggests that combined mechanisms act to prevent excessive activity of the CDX2 homeoprotein in stem/progenitor cells and hence the premature cell differentiation known to result from excessive CDX2 in the crypts. ${ }^{9}$

CDX2 has a role in the patterning of the intestine. ${ }^{38}$ Here, overexpressing miniCDX2 leads to extend the BG territory, which is accompanied by an increase of $P D X 1$, a homeobox gene of the paraHox cluster, like $C D X 2$, involved in $B G$ morphogenesis. ${ }^{29}$ This suggests that PDX1, together with the CDX2/miniCDX2 balance, participate in shaping the gastricintestinal boundary, in that the BG territory would depend on a low activity of CDX2 in the proximal small intestine, whereas miniCDX2 overexpression, through DNA-binding competition with CDX2, would allow extending the territory of these glands. In the long term, miniCDX2 overexpression in the BGs leads to brunneroma in which the production of EGF is increased and activates the EGFR in the overlaying crypts. Interestingly, these polyps exhibit gastric-type differentiation and even squamous stratified differentiation, but do not spontaneously progress in cancer, like most brunneroma in man. In line with this, the foregut-type heteroplasia developing in the cecum of $C d x 2^{+/-}$mice ${ }^{3}$ and the cecal gastric-type lesions generated by conditional $C d x 2$ knockout in the adult gut ${ }^{28}$ also fail to spontaneously progress to malignancy. Thus, the overexpression of the dominant-negative variant miniCDX2 and the loss of function of CDX2 do not represent oncogenic events per se. Yet, CDX2 has a tumor suppressor activity in the gut, as assessed by model studies in mice ${ }^{12,13}$ and observations made in colon cancer patients. ${ }^{10,39}$ Here, the data showing a modified CDX2/miniCDX2 ratio in favor of miniCDX2 in intestinal cancer open the possibility that miniCDX2, through its dominant-negative effect exerted on the tumor suppressor CDX2, may contribute to disease progression.

Here, we uncovered and characterized an original variant of the $C D X 2$ homeobox gene, which is a major player of intestinal morphogenesis, homeostasis and also involved in gut cancer. The production of this variant represents not only a novel level of regulation of this gene, but also a new way to fine-tune its biological activity through the versatile functions exerted by the truncated variant compared to the full-length homeoprotein. As in the case of another important transcription factor, SOX $9,{ }^{40}$ this study highlights the relevance of generating protein diversity through alternative splicing in the gut and its diseases.

\section{Materials and Methods}

Mice and generation of transgenic animals. Mice were handled according to the protocol approved by the Committee on the Ethics of Animal Experiments of the University of Strasbourg (CREMEAS, C2EA-35) under the permit number $\mathrm{AL} / 43 / 50 / 02 / 13$.

To generate jojo-Flag-miniCDX2 mice, the Flag-miniCDX2 sequence of plasmid pFlag-miniCDX2 (see below) was PCR-amplified using the primers AAAAGTC GACTACCATGGACTACAAAGACGATGA/AAAAGTCGACTCAGCCTGGAATTG $\overline{C T C}$ $\overline{T G C C G}$ containing each a Sall restriction site. The resulting Sall fragment was inserted in the Xhol site of the vector jojo $0^{41}$ to get plasmid jojo-Flag-miniCDX2. The 
eluted 8010 bp Sall fragment containing the transgene was injected in mouse eggs at the Mouse Clinic Institute (IIlkirch, France). Mice were PCR-genotyped with primers 39/hC2E3R (AGTCATAGCTGTCCCTCTTC/TCAGCCTGGAATTGCTCTGC).

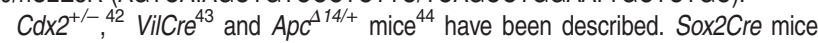
were obtained from The Jackson Laboratory (Charles River, L'Arbresle, France).

Cell lines and colony formation assays. Human colon cancer cell lines Caco2TC7 $,{ }^{45} \mathrm{HCT} 116,{ }^{46} \mathrm{SW} 480^{47}$ and $\mathrm{T} 84,{ }^{48}$ and human embryonic kidney cells HEK $293^{49}$ were grown as recommended. Colony formation assays were performed on HCT116 cells transfected with the indicated plasmids, as previously described. ${ }^{35}$

\section{Plasmids}

pFlag-CDX2 and pFlag-miniCDX2: RNA extracted from Caco2TC7 cells was subjected to RT-PCR using Platinum TaqPCRx DNA polymerase (Promega Inc., Madison, WI, USA) with the primers AAAAAAGCTTTACGTGAGCTACCTCCT GGACAAGG and AAAATCTAGATCAGCCTGGAATTGCTCTGC to amplify the human CDX2 coding sequence, and with the primers AAAAAAGCTTGTGAGGTC TGCTCCCGGACCCTCGC and AAAATCTAGATCAGCCTGGAATTGCTCTGC to amplify the miniCDX2 coding sequence. PCR fragments were inserted in pCRIITOPO (TA cloning kit, Life Technologies Invitrogen, Villebon sur Yvette, France), giving, respectively, PCRII-TOPO-CDX2 and PCRII-TOPO-miniCDX2. These plasmids were cut with HindIII and the fragments inserted in the HindllI site of pFlag-CMV2 (Sigma-Aldrich, St. Louis, MO, USA) to get the plasmids pFlag-CDX2 and pFlag-miniCDX2, respectively.

pATG1-ATG2 and pATG1m-ATG2: To construct pATG1-ATG2, the $5^{\prime}$-untranslated region of the mouse $C d x 2$ gene up to the codon $A T G_{2}$ was PCRamplified from pSL1190-9.4733 with the primers TTTTAAGCTTAAGGCCGCTGGC CTGGGACTCCGCGAITTTTCCATGGTGGCGAGGGACCCAGAGCAGA containing, respectively, HindllI and Ncol sites. The PCR fragment was cut with HindllI and $\mathrm{Ncol}$, and inserted in the corresponding sites of pGI3-control (Promega Inc.) to give pATG1-ATG2. pATG1m-ATG2 was derived from pATG1-ATG2 by site-directed mutagenesis using the Quick Change IIXL Site Directed Mutagenesis Kit (BD Biosciences, San Rose, CA, USA) with the primer TCAACGTTTGTCCCCAGACA CCTAGGTGAGGTCTGCTCTGGGT to change the ATG ${ }_{1}$ start codon into TAG.

$\bar{p} A \overline{T G} 1$ and pATG1m: Plasmid pATG1 containing the $5^{\prime}$-untranslated region of the murine $C d x 2$ gene up to the codon ATG $_{1}$ was constructed as above by PCR amplification with the primers TTTTAAGCTTAAGGCCGCTGGCCTGGGACTCCG CGA and TTTTCCATGGTGTCTGGGGACAAACGTTGT in which the sequence was changed for one nucleotide to create a Ncol cloning site. After insertion of the PCR fragment in the HindllI and Ncol sites of pGl3-control, the correct sequence was restored by point mutagenesis with the primer TTTGTCCCCAGACAGCATGG AAGACGCCAAAAACAT to give pATG1. pATG1m was derived from pATG1 by mutation with the primer TTTGTCCCCAGACACCTAGGAAGACGCCAAAAACAT to change the $A T G_{1}$ start codon into TAG.

eC2/1 and eC2/1m: The human CDX2 minigene overlapping the exon-1, the intron-1 and the fused exon-2/-3 and having the Flag coding sequence inserted before the Stop codon in the exon-3 was sequentially constructed using three overlapping fragments generated by PCR using Platinum TaqPCRx DNA Polymerase (Life Technologies Invitrogen): the fragment A and B were, respectively, amplified by PCR from human genomic DNA with the primers Af (GTTTA AACTTAAGCTTGCTCCGCACGCCAGCCTGT)/Ar (TCACAGGTCAGAGGTTCAGAG), and with the primers $\mathrm{Bf}$ (CTCTGAACCTCTGACCTGTGA)/Br (TCCGTGTACAC CACTCGATATTT); the fragment $C$ was amplified from pFlag-CDX2 DNA using the primers $\mathrm{Cf}$ (AGTGGTGTACACGGACCACC) and $\mathrm{Cr}$ (GCCCTCTAGACTCGAGTC ACTTGTCGTCATCGTCTTTGTAGTCCTGGGTGACGGTGGGGTTTA). Af contains 15 nucleotides complementary to the cloning site of the vector pcDNA4/TO, followed by 20 nucleotides complementary to the $5^{\prime}$ extremity of the CDX2 gene; Cr contains 15 nucleotides complementary to the cloning site of pcDNA4/TO, followed by a translation Stop codon, 24 nucleotides complementary to the Flag sequence and 20 nucleotides complementary to the CDX2 gene; the primers $\mathrm{Ar} / \mathrm{Bf}$ are partially complementary over 15 nucleotides, as the primers $\mathrm{Br} / \mathrm{Cf}$. The three PCR fragments $A, B$ and $C$ and the vector pcDNA4/TO cut with Hindlll and Xhol were purified by agarose gel electrophoresis, mixed and incubated with the In-Fusion HD Cloning kit (Clontech Laboratories, Inc., Mountain View, CA, USA). The resulting plasmid eC2I1 was verified by sequencing. eC211m was derived from eC2l1 using the primer ACCCTCGCCACCATGAACGTGAGCTACCTCC to change the GT splicing site into GA.

Other plasmids were: $\mathrm{pS} 33 \mathrm{~A}-\beta$-catenin encoding activated $\beta$-catenin, ${ }^{50} \mathrm{pcDNA3}$ TCF4E-myc encoding Myc-tagged Tcf4, ${ }^{51}$ the reporter plasmids TOPFlash, ${ }^{52}$
pSI-Luc, ${ }^{53}$ pHis-SRp30c and pHis-ASF/SF $2,{ }^{54}$ and commercially-available pEGFP (Clontech Laboratories Inc.), pGI3-basic, pGI3-control, pRL-Null (Promega Inc.) and pCMV-Flag2 (Sigma-Aldrich).

Antibodies. The antibody against the miniCDX2 protein was obtained by immunizing rabbits with the synthetic peptide MVRSAPGPSPP-cystein coupled to $\mathrm{KLH}$ (Eurogentech SA, Seraing, Belgium). C2T anti-peptide antibody was purified by affinity chromatography. The peptide was reduced by TCEP (Tris-(2-carboxyethyl) phosphine) using Immobilized TCEP-Disulfide Reducing gel (Pierce Biotechnology, Rockford, IL, USA). Reduced peptide of $2.5 \mathrm{mg}$ were incubated with $2 \mathrm{ml}$ sulfonylagarose (SulfoLink Trial kit, Pierce Biotechnology) during $1 \mathrm{~h}$ and the affinity gel was equilibrated in Tris $50 \mathrm{mM} / \mathrm{NaCl} 0.15 \mathrm{M} \mathrm{pH} 7.3$ buffer. Rabbit immune serum of $2 \mathrm{ml}$ were passed through the affinity column, washed with Tris $50 \mathrm{mM} / \mathrm{NaCl} 0.15-0.5 \mathrm{M}$ $\mathrm{pH}$ 7.3. Specific immunoglobulins were eluted with glycine $0.2 \mathrm{M} \mathrm{pH} 2.7$ and neutralized with $50 \mu \mathrm{l}$ Tris buffer $1 \mathrm{M} \mathrm{pH}$ 8. The C2T antibody raised against the Nter part of miniCDX2 was used at $15 \mu \mathrm{g} / \mathrm{ml}$.

Commercial antibodies were against: actin (clone C4, Millipore, St. Quentin-enYvelines, France), ASF/SF2 (sc-33652, Santa Cruz Biotechnology Inc., Santa Cruz, CA, USA), $\beta$-catenin (mouse clone 14, BD Transduction Lab, San Rose, CA, USA), CDX2 (mouse clone 392M, BioGenex, San Ramon, CA, USA), Claudin-18 (Life Technologies Invitrogen), Flag (mouse clone M2, Sigma-Aldrich), GFP (BD Biosciences, Franklin Lakes, NJ, USA), HA (clone 3F10, Roche Applied Science, Meylan, France), KU70 (rabbit clone EPR4027, Abcam, Cambridge, MA, USA), KU80 (rabbit clone EPR3468, Abcam), Myc (mouse clone 9E10, Santa Cruz Biotechnology Inc.), p27KIP1 (mouse clone 57, BD Transduction Lab), PDX1 (rabbit, Abcam ab47267), phospho-EGFR (Cell Signaling Technology, Ozyme, Montigny-leBretonneux, France), phospho-histone-3 (Ser10 mitosis marker, Millipore Upstate, Saint-Quentin Yvelines, France) and SRp30c (sc-134046, Santa Cruz Biotechnology Inc.).

Cellular RNA preparation and in vitro RNA transcription. RNA was prepared using TRI Reagent (Life Technologies Ambio, Villebon sur Yvette, France) and treated with DNAse (RQ1 RNAse-free DNAse, Promega Inc.).

For in vitro transcription of capped and uncapped RNA, DNA templates were amplified using Platinum TaqPCRx DNA polymerase from the plasmids pATG1, pATG1-ATG2, pATG1m-ATG2 and pGL3-control with Tption-f/Tption-r (TTTTTTTTT TTAATACGACTCACTATAGGGAGAAGGCCTAGGCTTTTGCAAAAAGCTT/(T) ${ }_{42}$ CACT GCATTCTAGTTGTG) containing, respectively, the T7 promoter and polydT sequences. After purification (QIAquick PCR Purification kit, Qiagen, Venio, The Netherlands), $1 \mu \mathrm{g}$ of each PCR fragment was in vitro transcribed with or without $m 7 \mathrm{G}\left(5^{\prime}\right) \operatorname{ppp}\left(5^{\prime}\right) \mathrm{G}$ RNA Cap (S1404S, BioLabs, Ipswich, MA, USA) in the ratio of 1 GTP/4 GTPcap, in $25 \mu$ l using HiScribe T7 in vitro Transcription kit (BioLabs). After treatment with DNAse (RQ1 RNAse-free DNAse, Promega Inc.), RNAs were precipitated in NH4-acetate $2.5 \mathrm{M}$ on ice, washed in $70 \%$ ethanol and solubilized in $5 \mu \mathrm{l}$ RNAsefree water.

RNAseq analysis. RNA integrity was checked on nano RNA chips with a Bioanalyser 2100 (Agilent Technologies, Santa Clara, CA, USA) and no sign of RNA degradation was detected. Total RNA of $1 \mu \mathrm{g}$ was used to construct the mRNAseq libraries with Illumina's TruSeq RNA sample kit following manufacturer's instructions (Illumina, San Diego, CA, USA). Libraries were validated and quantified on DNA1000 chips and a Bioanalyser 2100 (Agilent Technologies, Les Ulis, France). Paired-end reads of $50 \mathrm{bp}$ were obtained with a HiSeq1000 by multiplexing three libraries on one lane. Demultiplexing and generation of raw fastq files were performed with CASAVA v1.7. Mapping against the reference mouse genome GRCm38 was performed with tophat 2.0.11 and bowtie2 using the options -b2sensitive -a 5 -p 5 -library-type fr-unstranded $-r \quad 180$-mate-std-dev 80. Quantification of the reads was performed with HTSeq and DESeq2 using the reference gene annotation from ensembl v75. Genes with $>10$ normalized reads in the three replicates as given in DESeq2 were considered as significantly detected. De novo transcripts assembly was performed with cufflinks v2.2.1 with the options - $u$-library-type fr-firststrand, and providing the reference genome fasta file and gene annotation from ensembl v75. Search of novel exon-exon jonctions was made with cuffcompare and consensus novel junctions found in all biological triplicates obtained with bedtools. The resulting bed file was then imported in $\mathrm{R}$ as a Grange object for gene annotation.

Northern blots, RT-PCR and RT-qPCR. PolyA+ RNA (PolyATract System, Promega Inc.) was separated by electrophoresis on $2 \%$ agarose/2 $\mathrm{M}$ formaldehyde 
gels (10 $\mu \mathrm{g}$ polyadenylated RNA/lane) and transferred to nylon membranes. Hybridization and revelation was performed as described ${ }^{55}$ with $100 \mathrm{ng} / \mathrm{ml}$ of DIGlabeled antisense cRNA probe. This probe overlapped the $3^{\prime}$-untranslated region of the exon-3 of the human CDX2 gene, and was in vitro transcribed by T7 RNA polymerase from plasmid pCRII-TOPO-miniCDX2 linearized with BsrGI. A DIGlabeled human GAPDH probe was used for normalization.

Reverse transcription was performed with $2 \mu \mathrm{g}$ RNA using SuperScript II Reverse Transcriptase (Life Technologies, Invitrogen) and Oligo(dT)12-18 primer (Invitrogen).

RT-PCR used 1/10th of the RT solution. Concomitant amplification of the two splicing forms of the human $C D X 2$ gene was performed with primers CDX21F/CCR (GCAGCCTCCAGCGTCGGTC/TCAGCCTGGAATTGCTCTGC) for 35 cycles (30 s at $95{ }^{\circ} \mathrm{C}, 30 \mathrm{~s}$ at $55^{\circ} \mathrm{C}$ and $1.5 \mathrm{~min}$ at $68^{\circ} \mathrm{C}$ ). Specific amplification of the mouse CDX2 mRNA and miniCDX2 mRNA, respectively, used the primer pairs mhC2Flf/ mhC2E3r (CCCTCGCCACCATGTACG/CTCTGCGGTTCTGAAACCA) and mhC2Tqf (CCCTCGCCACCATTGAAAA)/mhC2E3r.

For RT-qPCR, $2 \mu \mathrm{g}$ of mouse intestinal RNA, of RNA from human colon biopsies or from human colon cancer Caco2TC7 cells were reverse transcribed in $20 \mu$ l using the AMV Reverse Transcriptase kit (Life Technologies, Invitrogen) and oligo-dT. RT-qPCR was performed on $1 \mu \mathrm{l}$ of RT reaction in $20 \mu \mathrm{l}$ using Syber Green PCR Master Mix (Life Technologies Applied Biosystems, Villebon sur Yvette, France) and 7500 Real-time PCR System (Life Technologies Applied Biosystems). For mouse RNA, the cycles were $95^{\circ} \mathrm{C} 15 \mathrm{~s}, 57^{\circ} \mathrm{C} 1 \mathrm{~min}$, with the primers: CCCTCGCCACCATTGAAAA/ GTGATGTATCGACTAAAGTG for miniCDX2, CTAGGAAGCCAAGTGAAAAC/GTG ATGTATCGACTAAAGTG for CDX2 and CCCCACAACTCTTCCATTCT/GCAGG AGTGATAGGGGTCAT for TBP. For Caco2TC7 cell RNA, the cycles were $95^{\circ} \mathrm{C}$ $15 \mathrm{~s}, 68^{\circ} \mathrm{C} 1 \mathrm{~min}$ with the primers: CCCTCGCCACCATTGAAAA/TGCCTCTCAGAG AGCCCCAGCGTGG for miniCDX2, CTCGGCAGCCAAGTGAAAAC/GTGATGTA GCGACTGTAGTG for CDX2 and TGCACAGGAGCCAAGAGTGAA/CACATCACAGC TCCCCACCA for TBP.

Quantification of the SI, MMP7, P27KIP1, EGF and TBP transcripts was performed by RT-qPCR using diluted RT products (5-10x) mixed with TaqMan Master Mix, genespecific TaqMan probe and the primers sets (TaqMan Gene Expression assays, Life Technologies Applied Biosystems): SI, Hs00356112_m1; MMP7, Hs01042795_m1; P27KIP1, Hs01597588_m1; EGF, Hs01099999_m1; TBP, Hs99999910_m1.

Analysis of the results (triplicates) was performed with the 7500 software v2.0.1 (Life Technologies Applied Biosystems) using the relative $\Delta \Delta \mathrm{Ct}$ quantification method.

DNA and RNA transfections and luciferase assays. Plasmid DNA transfection was performed during $48 \mathrm{~h}$ with JetPEI (Polyplus Transfection, IIIkirch, France). RNA transfection was performed for $3 \mathrm{~h}$ with $100-500 \mathrm{ng}$ of in vitrotranscribed RNA (see below) using 0.25-1.25 $\mu \mathrm{l}$ TransIT mRNA and 0.375-1.875 $\mu \mathrm{l}$ of boost (Mirus Bio, Madison, WI, USA) on cells at $80 \%$ confluence. Firefly and Renilla luciferase activities were measured at least three times in triplicate using a dual reporter luciferase assay (Promega Inc.) in cell extracts prepared $48 \mathrm{~h}$ after DNA transfection or $3 \mathrm{~h}$ after RNA transfection. Means are given \pm S.D.

Immunostaining. Immunochemical staining and/or immunofluorescence detection were performed on paraffin-embedded tissue sections. ${ }^{33}$ Histochemical staining used the Vectastain $\mathrm{ABC}$ kit (Vector Labs, Peterborough, UK) and DAB $0.3 \mathrm{mg} / \mathrm{ml} / \mathrm{H}_{2} \mathrm{O}_{2} \quad 0.03 \%$. Double-immunofluorescence staining used secondary Alexa488-labeled anti-mouse antibody and Alexa688-labeled anti-rabbit antibody (Life Technologies Invitrogen) coupled to fluorescence amplification with the TSA Fluorescent Plus System (Perkin Elmer, Waltham, MA, USA).

Co-immunoprecipitations and western blots. Transfected cells were lysed in buffer containing $50 \mathrm{mM}$ Tris buffer pH 7.4, $150 \mathrm{mM} \mathrm{NaCl}, 1 \mathrm{mM}$ EDTA and protease inhibitor cocktail (Roche Diagnostic, Mannheim, Germany). The buffer additionally contained $1 \%$ Triton $\mathrm{X}-100$ for $\beta$-catenin and Flag immunoprecipitation, or $1 \%$ Triton X-100 and $1 \%$ NP-40 for ASF/SF2 immunoprecipitation, or $1 \%$ NP-40 for HA immunoprecipitation. Lysates were cleared by centrifugation at $12000 \times g$ for $20 \mathrm{~min}$ at $4{ }^{\circ} \mathrm{C}$, and the protein extracts were then treated with $1 \mu \mathrm{l}$ of $0.1 \mathrm{mg} / \mathrm{ml}$ $\mathrm{RNaseA}$ for $30 \mathrm{~min}$ on ice when indicated. Immunoprecipitation was performed using $1 \mathrm{mg}$ of lysate supernatant incubated overnight at $4^{\circ} \mathrm{C}$ with gentle rocking in $1 \mathrm{ml}$ of lysis buffer with the appropriate antibody: anti-Flag antibody $(5 \mu \mathrm{g}$ of clone M2, Sigma-Aldrich), anti- $\beta$-catenin antibody ( $1 \mu \mathrm{g}$ of clone $14, \mathrm{BD}$ Biosciences), anti-HA antibody ( $20 \mu \mathrm{l}$ of agarose beads coupled to clone $3 \mathrm{~F} 10$, Roche Applied Science) and anti-ASF/SF2 antibody ( $3 \mu \mathrm{g}$ of sc-33652, Santa Cruz Biotechnology Inc.). After adding $25 \mu$ l of Protein-G-agarose beads (Roche Applied
Science) for $2 \mathrm{~h}$ at $4{ }^{\circ} \mathrm{C}$, the beads were washed in lysis buffer. The immunoprecipitated material was eluted using SDS-PAGE sample loading buffer and analyzed by western blots performed as described. ${ }^{35}$

Chromatin immunoprecipitations. Transfected HCT116 cells were fixed with $1 \%$ (v/v) formaldehyde for $10 \mathrm{~min}$ at RT and quenched with $0.125 \mathrm{M}$ glycine for $5 \mathrm{~min}$. ChIPs were carried out using the EZ-Magna ChIP G Chromatin Immunoprecipitation kit (Upstate Millipore, Billerica, MA, USA). Sheared cross-linked chromatin from $\sim 10^{6}$ cells was incubated overnight at $4{ }^{\circ} \mathrm{C}$ with $1 \mu \mathrm{g}$ of normal mouse IgG (Upstate Millipore) or the appropriate antibody. Input corresponds to non-immunoprecipitated sheared cross-linked chromatin from $10^{5}$ cells. PCR analysis was performed with $1 / 25$ th of immunoprecipitated DNA as template. The primers used for PCR amplification of three human targets of the CDX2 protein were: SI promoter GGCTGGTAAGGGTGCAATAA/GCCTGTTCTCTTTGCTATGTTG; Muc2 promoter: GTGTGTTGGCATTCAGGCTA/AGGGTAGGAGGGCAGGAAG; Li-cad promoter: TTTCAAAAGCAGGCACAGCC/AGTGGTCGAGACTCTTGCTAC.

In vitro DNA repair assays. Cell-free extracts were prepared as follows. A total of $3 \times 10^{7}$ transfected cells were washed in cold PBS, suspended in four packed cell volumes of cold hypotonic lysis buffer $(10 \mathrm{mM}$ Tris- $\mathrm{HCl} \mathrm{pH} \mathrm{7.6,1 \textrm {mM }}$ DTT, $5 \mathrm{mM} \mathrm{MgCl} 2,1 \mathrm{mM}$ EDTA and protease inhibitors), incubated for $40 \mathrm{~min}$ on ice and disrupted using a Dounce homogenizer (40 strokes). Sucrose was added to reach $250 \mathrm{mM}$. Extracts were centrifuged $10 \mathrm{~min}$ at $1000 \times \mathrm{g}$ to discard cell debris and washed again in hypotonic lysis buffer containing $250 \mathrm{mM}$ sucrose. Nuclear pellet was then suspended in two volumes of nuclear extraction buffer (20 mM Tris-HCl pH 7.6, 1 mM DTT, 2 mM EDTA, 20\% glycerol, $500 \mathrm{mM}$ $\mathrm{NaCl}$ and protease inhibitors) and incubated for $30 \mathrm{~min}$ on ice. Nuclear extracts were clarified by 30 min centrifugation at $21000 \times g$ and dialyzed overnight against $20 \mathrm{mM}$ Tris-HCl pH 7.6, $1 \mathrm{mM}$ EDTA, $1 \mathrm{mM} \mathrm{DTT,} 20 \%$ glycerol, $25 \mathrm{mM} \mathrm{NaCl}$ and $0.2 \mathrm{mM}$ PMSF

End-joining reaction was performed in $30 \mu \mathrm{l}$ by incubating $50 \mathrm{ng}$ of BamHllinearized pcDNA3 plasmid with $5 \mathrm{mg}$ of nuclear extract for $1 \mathrm{~h}$ at $25^{\circ} \mathrm{C}$ in $50 \mathrm{mM}$ Tris- $\mathrm{HCl} \mathrm{pH} \mathrm{7.6,} 5 \mathrm{mM} \mathrm{MgCl}$, 1 mM ATP, $1 \mathrm{mM}$ DTT, $50 \mathrm{mM}$ dNTP, $80 \mathrm{mM} \mathrm{NaCl}$ and protease inhibitors. Reaction was stopped by RNAse treatment $(0.25 \mathrm{mg} / \mathrm{ml}$ of RNAse for $10 \mathrm{~min}$ at $37^{\circ} \mathrm{C}$ ), followed by proteinase $\mathrm{K}(0.5 \% \mathrm{SDS}, 50 \mathrm{mM}$ EDTA and $1 \mathrm{mg} / \mathrm{ml}$ of proteinase $\mathrm{K}$ at $37^{\circ} \mathrm{C}$ for $1 \mathrm{~h}$ ). DNA was purified by phenol and chloroform extraction, recovered by ethanol precipitation and analyzed by electrophoresis on a $0.85 \%$ agarose gel.

Statistical analyses. Experiments were performed in at least three independent biological replicates in triplicates. $P$-value calculation indicated significant differences tested by the Wilcoxon-Mann-Whitney test.

\section{Conflict of Interest}

The authors declare no conflict of interest.

Acknowledgements. This work was supported by the INSERM (France) and the Fondation ARC (grants 3759 and 4872). CB, AN and CS were funded by the MRES (France). CB and CS were further supported by the Ligue Contre le Cancer, and TS by the Worldwide Cancer Research, UK (08-0199). We thank Dr. MA Birling and the Mouse Clinic Institute for generating the jojo-Flag-miniCdx2 mice, Professor MP Chenard (CHU de Strasbourg, France) for pathological evaluation of the mice, Dr. S Robine (CNRS, UMR 144, Institut Curie, Paris) for the VilCre mice, Professor B Chabot (Université de Sherbrooke) for the plasmids pHis-SRp30c and pHis-ASF/ SF2, and Dr. JF Launay (Inserm U682, Strasbourg) for help in C2T antibody purification. The data described here have been deposited in the GEO data base under the access code GSE89992. The Genbank accession number of human miniCDX2 is KJ531444.

1. Clevers H. The intestinal crypt, a prototype stem cell compartment. Cell 2013; 154: 274-284.

2. Li H, Jasper H. Gastrointestinal stem cells in health and disease: from flies to humans. Dis Model Mech 2016; 9: 487-499.

3. Beck F, Chawengsaksophak K, Waring P, Playford RJ, Furness JB. Reprogramming of intestinal differentiation and intercalary regeneration in cdx2 mutant mice. Proc Natl Acad SC USA 1999; 96: 7318-7323.

4. Gao N, White P, Kaestner $\mathrm{KH}$. Establishment of intestinal identity and epithelialmesenchymal signaling by Cdx2. Dev Cell 2009; 16: 588-599. 
5. San Roman AK, Tovaglieri A, Breault DT, Shivdasani RA. Distinct processes and transcriptional targets underlie CDX2 requirements in intestinal stem cells and differentiated villus cells. Stem Cell Rep 2015; 5: 673-681.

6. Simmini S, Bialecka M, Huch M, Kester L, van de Wetering M, Sato T et al. Transformation of intestinal stem cells into gastric stem cells on loss of transcription factor Cdx2. Nat Commun 2014; 5 : 5728.

7. Stringer EJ, Duluc I, Saandi T, Davidson I, Bialecka M, Sato T et al. Cdx2 determines the fate of postnatal intestinal endoderm. Dev Camb Engl 2012; 139: 465-474.

8. Verzi MP, Shin H, Ho L-L, Liu XS, Shivdasani RA. Essential and redundant functions of caudal family proteins in activating adult intestinal genes. Mol Cell Biol 2011; 31: 2026-2039.

9. Crissey MAS, Guo R-J, Funakoshi S, Kong J, Liu J, Lynch JP. Cdx2 levels modulate intestinal epithelium maturity and Paneth cell development. Gastroenterology 2011; 140 517-528.e8.

10. Bae JM, Lee TH, Cho N-Y, Kim T-Y, Kang GH. Loss of CDX2 expression is associated with poor prognosis in colorectal cancer patients. World $J$ Gastroenterol 2015; 21 : 1457-1467.

11. De Sousa E, Melo F, Wang X, Jansen M, Fessler E, Trinh A et al. Poor-prognosis colon cancer is defined by a molecularly distinct subtype and develops from serrated precursor lesions. Nat Med 2013; 19: 614-618.

12. Aoki K, Tamai Y, Horiike S, Oshima M, Taketo MM. Colonic polyposis caused by mTORmediated chromosomal instability in Apc+/Delta716 Cdx2+/- compound mutant mice. Nat Genet 2003; 35: 323-330.

13. Bonhomme C, Duluc I, Martin E, Chawengsaksophak K, Chenard M-P, Kedinger M et al. The $\mathrm{Cdx} 2$ homeobox gene has a tumour suppressor function in the distal colon in addition to a homeotic role during gut development. Gut 2003; 52: 1465-1471.

14. Gross I, Duluc I, Benameur T, Calon A, Martin E, Brabletz T et al. The intestine-specific homeobox gene $\mathrm{Cdx2}$ decreases mobility and antagonizes dissemination of colon cancer cells. Oncogene 2008; 27: 107-115.

15. Hryniuk A, Grainger S, Savory JGA, Lohnes D. Cdx1 and Cdx2 function as tumor suppressors. J Biol Chem 2014; 289: 33343-33354.

16. Kelemen $\mathrm{O}$, Convertini $\mathrm{P}$, Zhang Z, Wen $\mathrm{Y}$, Shen $\mathrm{M}$, Falaleeva $\mathrm{M}$ et al. Function of alternative splicing. Gene 2013; 514: 1-30.

17. Nilsen TW, Graveley BR. Expansion of the eukaryotic proteome by alternative splicing Nature 2010; 463: 457-463.

18. Chepelev I, Chen X. Alternative splicing switching in stem cell lineages. Front Biol 2013; 8 50-59.

19. Cieply B, Carstens RP. Functional roles of alternative splicing factors in human disease. Wiley Interdiscip Rev RNA 2015; 6: 311-326.

20. Muñoz J, Stange DE, Schepers AG, van de Wetering M, Koo B-K, Itzkovitz S et al. The Lgr5 intestinal stem cell signature: robust expression of proposed quiescent ' +4 ' cell markers. EMBO J 2012; 31: 3079-3091.

21. Kozak M. An analysis of 5'-noncoding sequences from 699 vertebrate messenger RNAs. Nucleic Acids Res 1987; 15: 8125-8148.

22. Kozak M. Pushing the limits of the scanning mechanism for initiation of translation. Gene 2002; 299: 1-34

23. Stoneley M, Spencer JP, Wright SC. An internal ribosome entry segment in the 5' untranslated region of the mnt gene. Oncogene 2001; 20: 893-897.

24. Freund JN, Duluc I, Reimund JM, Gross I, Domon-Dell C. Extending the functions of the homeotic transcription factor $\mathrm{Cdx} 2$ in the digestive system through nontranscriptional activities. World J Gastroenterol 2015; 21: 1436-1443.

25. Guo R-J, Funakoshi S, Lee HH, Kong J, Lynch JP. The intestine-specific transcription factor Cdx2 inhibits beta-catenin/TCF transcriptional activity by disrupting the beta-catenin-TCF protein complex. Carcinogenesis 2010; 31: 159-166.

26. Aoki K, Kakizaki F, Sakashita H, Manabe T, Aoki M, Taketo MM. Suppression of colonic polyposis by homeoprotein CDX2 through its nontranscriptional function that stabilizes p27Kip1. Cancer Res 2011; 71: 593-602.

27. Renouf B, Soret C, Saandi T, Delalande F, Martin E, Vanier M et al. Cdx2 homeoprotein inhibits non-homologous end joining in colon cancer but not in leukemia cells. Nucleic Acids Res 2012; 40: 3456-3469.

28. Stringer EJ, Duluc I, Saandi T, Davidson I, Bialecka M, Sato T et al. Cdx2 determines the fate of postnatal intestinal endoderm. Development 2012; 139: 465-474.

29. Offield MF, Jetton TL, Labosky PA, Ray M, Stein RW, Magnuson MA et al. PDX-1 is required for pancreatic outgrowth and differentiation of the rostral duodenum. Development 1996; 122: $983-995$
30. Powell AE, Wang Y, Li Y, Poulin EJ, Means AL, Washington MK et al. The pan-ErbB negative regulator Lrig1 is an intestinal stem cell marker that functions as a tumor suppressor. Cell 2012; 149: 146-158.

31. Flores MV, Hall CJ, Davidson AJ, Singh PP, Mahagaonkar AA, Zon LI et al. Intestinal differentiation in zebrafish requires $\mathrm{Cdx} 1 \mathrm{~b}$, a functional equivalent of mammalian $\mathrm{Cdx2}$. Gastroenterology 2008; 135: 1665-1675.

32. Wethmar K, Smink JJ, Leutz A. Upstream open reading frames: molecular switches in (patho)physiology. BioEssays News Rev Mol Cell Dev Biol 2010; 32: 885-893.

33. Benahmed F, Gross I, Gaunt SJ, Beck F, Jehan F, Domon-Dell C et al. Multiple regulatory regions control the complex expression pattern of the mouse Cdx2 homeobox gene. Gastroenterology 2008; 135: 1238-1247.

34. Saandi T, Baraille F, Derbal-Wolfrom L, Cattin AL, Benahmed F, Martin E et al. Regulation of the tumor suppressor homeogene Cdx2 by HNF4alpha in intestinal cancer. Oncogene 2013; 32: $3782-3788$

35. Gross I, Lhermitte B, Domon-Dell C, Duluc I, Martin E, Gaiddon C et al. Phosphorylation of the homeotic tumor suppressor $\mathrm{Cdx2}$ mediates its ubiquitin-dependent proteasome degradation. Oncogene 2005; 24: 7955-7963.

36. Rings EH, Boudreau F, Taylor JK, Moffett J, Suh ER, Traber PG. Phosphorylation of the serine 60 residue within the $\mathrm{cdx} 2$ activation domain mediates its transactivation capacity. Gastroenterology 2001; 121: 1437-1450.

37. Witek ME, Snook AE, Lin JE, Blomain ES, Xiang B, Magee MS et al. A novel CDX2 isoform regulates alternative splicing. PLOS ONE 2014; 9: e104293.

38. Grainger S, Savory JGA, Lohnes D. Cdx2 regulates patterning of the intestinal epithelium. Dev Biol 2010; 339: 155-165.

39. Dalerba $P$, Sahoo D, Paik $S$, Guo X, Yothers $G$, Song $N$ et al. CDX2 as a prognostic biomarker in stage II and stage III colon cancer. N Engl J Med 2016; 374: 211-222.

40. Abdel-Samad R, Zalzali H, Rammah C, Giraud J, Naudin C, Dupasquier S et al. MiniSOX9, a dominant-negative variant in colon cancer cells. Oncogene 2011; 30: 2493-2503.

41. Collombat P, Hecksher-Sorensen J, Krull J, Berger J, Riedel D, Herrera PL et al. Embryonic endocrine pancreas and mature beta cells acquire alpha and PP cell phenotypes upon Arx misexpression. J Clin Invest 2007; 117: 961-970.

42. Chawengsaksophak K, James R, Hammond VE, Kontgen F, Beck F. Homeosis and intestinal tumours in Cdx2 mutant mice. Nature 1997; 385: 84-87.

43. El Marjou F, Janssen KP, Chang BH, Li M, Hindie V, Chan L et al. Tissue-specific and inducible Cre-mediated recombination in the gut epithelium. Genesis 2004; 39: 186-193.

44. Colnot S, Niwa-Kawakita M, Hamard G, Godard C, Le Plenier S, Houbron C et al. Colorectal cancers in a new mouse model of familial adenomatous polyposis: influence of genetic and environmental modifiers. Lab Invest 2004; 84: 1619-1630.

45. Chantret I, Rodolosse A, Barbat A, Dussaulx E, Brot-Laroche E, Zweibaum A et al. Differential expression of sucrase-isomaltase in clones isolated from early and late passages of the cell line Caco-2: evidence for glucose-dependent negative regulation. J Cell Sci 1994; 107: 213-225.

46. Brattain MG, Fine WD, Khaled FM, Thompson J, Brattain DE. Heterogeneity of malignant cells from a human colonic carcinoma. Cancer Res 1981; 41: 1751-1756.

47. Leibovitz A, Stinson JC, McCombs WB III, McCoy CE, Mazur KC, Mabry ND. Classification of human colorectal adenocarcinoma cell lines. Cancer Res 1976; 36: 4562-4569.

48. Murakami $\mathrm{H}$, Masui $\mathrm{H}$. Hormonal control of human colon carcinoma cell growth in serum-free medium. Proc Natl Acad Sci USA 1980; 77: 3464-3468.

49. Graham FL, Smiley J, Russell WC, Nairn R. Characteristics of a human cell line transformed by DNA from human adenovirus type 5. J Gen Virol 1977; 36: 59-74.

50. Aberle H, Bauer A, Stappert J, Kispert A, Kemler R. Beta-catenin is a target for the ubiquitinproteasome pathway. EMBO J 1997; 16: 3797-3804.

51. Tetsu O, McCormick F. Beta-catenin regulates expression of cyclin D1 in colon carcinoma cells. Nature 1999; 398: 422-426.

52. van de Wetering M, Cavallo R, Dooijes D, van Beest M, van Es J, Loureiro J et al. Armadillo coactivates transcription driven by the product of the Drosophila segment polarity gene dTCF. Cell 1997; 88: 789-799.

53. Rodolosse A, Chantret I, Lacasa M, Chevalier G, Zweibaum A, Swallow D et al. A limited upstream region of the human sucrase-isomaltase gene confers glucose-regulated expression on a heterologous gene. Biochem J 1996; 315: 301-306.

54. Paradis C, Cloutier P, Shkreta L, Toutant J, Klarskov K, Chabot B. hnRNP I/PTB can antagonize the splicing repressor activity of SRp30c. RNA 2007; 13: 1287-1300.

55. Lefebvre O, Sorokin L, Kedinger M, Simon-Assmann P. Developmental expression and cellular origin of the laminin alpha2, alpha4, and alpha5 chains in the intestine. Dev Biol 1999; 210: 135-150.

\section{Supplementary Information accompanies this paper on Cell Death and Differentiation website (http://www.nature.com/cdd)}

